\title{
Supplementary information for Prediction of
}

\section{Protein-Ligand Binding Poses via a \\ Combination of Induced Fit Docking and Metadynamics Simulations}

Anthony J. Clark, ${ }^{\dagger}$ Pratyush Tiwary, ${ }^{* \dagger} \dagger$ Ken Borrelli, ${ }^{\ddagger}$ Shulu Feng, ${ }^{\ddagger}$ Edward B. Miller, ${ }^{\ddagger}$ Robert Abel, ${ }^{\ddagger}$ Richard A. Friesner, ${ }^{\dagger}$ and B. J. Berne ${ }^{\dagger}$

$\dagger$ †epartment of Chemistry, Columbia University, New York 10027, United States $\ddagger$ Schrödinger, Inc., 120 West 45th Street, New York, New York 10036, United States

E-mail: pt2399@columbia.edu

\section{Rigid Receptor Docking Results}

To demonstrate the requirement for IFD over RRD for the cases in our test set we performed RRD docking with Glide for all cases in the set. Table 1 summarizes the results for the top five poses generated (in some cases Glide does not generate 5 different poses). In the vast majority of cases RRD fails to produce a good pose in the top 5. In a handful of other cases (6 in all), Glide produces a sub- $2 \AA$ pose in the top 5 , and in only five of the 42 cases does Glide produce a sub- $2 \AA$ top scoring pose. Even for the handful of cases for which RRD can produce a pose meeting our criteria, the IFD data set remains a valid testing case for the ability of metadynamics to pick out a good pose out of a set of supplied poses, as there is no reason to expect a correlation between the requirement for re-arrangements of receptor atoms 
to generate a good pose and the ability of metadynamics to measure the relative stability of the poses generated by docking. Since the docking stage success rate sets an upper limit on the success rate of the overall protocol, metadynamics combined with Glide docking alone on this set would be guaranteed to perform extremely poorly.

\section{Alternate CV}

In parallel to the work described in this publication, a slight modification of the algorithm was developed that achieved comparable results. This is being presented here both for completeness and to demonstrate the robustness of the methodology. The primary difference between the two algorithms is the choice of collective variable. Instead of using the aligned RMSD of the ligand and a few anchor atoms on the protein to bias the metadynamics as presented in the publication, an RMSD collective variable was employed that aligned the binding sites of each frame in the trajectory before calculating an in-place RMSD of the ligand heavy atoms. The binding site was defined as the set of protein residues for which any atom was within 3 angstroms of the ligand in any of the poses to evaluate. The carbon-alphas of the binding site residues were then aligned to those of the first frame of the production-phase of the metadynamics trajectory before calculating the heavy atom RMSD to the ligand conformation in the first frame. Like the collective variable presented in the publication this one tracks the deviations in the position and conformation of the ligand in the binding site from the starting pose and should be applicable to most systems where a protein is binding to a small molecule.

There was also a slight difference both in the way the interaction persistence score was calculated and with how it was combined with the stability score. The interaction persistence score in this variant of the method included both hydrogen bond and Pi-Pi stacking, equally weighted, but otherwise was equivalent to the interaction persistence score presented in the publication. The elimination of anchor atoms in the collective variable, and therefore the 
Table 1: Full results of RRD using Glide are shown. Values given are RMSDs to native in A. Poses are sorted by Glide docking score.

\begin{tabular}{|c|c|c|c|c|c|c|c|}
\hline Case & Receptor PDB & Ligand PDB & Pose 1 & Pose 2 & Pose 3 & Pose 4 & Pose 5 \\
\hline alr2 & 1iei & $2 \mathrm{fz} 8$ & 2.26 & 10.43 & 10.15 & 10.36 & 8.52 \\
\hline alr2 & $2 \mathrm{fzd}$ & 1iei & 6.61 & 6.66 & 6.68 & 6.59 & 6.65 \\
\hline alr2 & $2 \mathrm{fzd}$ & $1 \mathrm{t} 40$ & 4.44 & 9.01 & 9.11 & 8.80 & 8.73 \\
\hline alr2 & $2 \mathrm{fzd}$ & $1 z 3 n$ & 8.34 & 8.38 & 8.70 & 8.28 & - \\
\hline alr2 & $2 \mathrm{fzd}$ & $2 \mathrm{hvn}$ & 6.97 & 9.68 & 9.42 & 9.27 & 9.98 \\
\hline alr2 & $2 \mathrm{fzd}$ & $2 \mathrm{ikg}$ & 3.01 & 5.15 & 5.10 & 4.94 & 5.33 \\
\hline cdk2 & $1 p x j$ & 1 jvp & 5.38 & 5.56 & 7.55 & 8.66 & - \\
\hline cdk2 & $1 p x j$ & 1ke8 & 7.12 & 7.68 & 7.20 & 10.69 & - \\
\hline cdk2 & $1 \mathrm{pxj}$ & 1 pxo & 5.81 & 6.10 & 6.83 & 5.80 & 6.69 \\
\hline cdk2 & $1 p x j$ & $2 \mathrm{aOC}$ & 6.58 & 8.39 & 7.30 & 7.71 & 8.60 \\
\hline cdk2 & $1 p x j$ & $2 \mathrm{btr}$ & 5.57 & 3.28 & 5.73 & 7.71 & 5.61 \\
\hline cdk2 & $1 \mathrm{pxj}$ & $2 \mathrm{bts}$ & 7.58 & 8.39 & 8.45 & 7.59 & 8.70 \\
\hline cdk2 & $1 \mathrm{pxj}$ & $2 \mathrm{c} 6 \mathrm{~m}$ & 2.43 & 2.49 & 2.05 & 2.01 & 9.35 \\
\hline cdk2 & $1 \mathrm{wcc}$ & 1oit & 5.31 & 7.58 & 5.28 & 5.08 & - \\
\hline cdk2 & 1wcc & $2 \mathrm{~b} 52$ & 5.61 & 6.76 & 7.20 & 9.18 & - \\
\hline cdk2 & 1wcc & 2bhe & 4.73 & 6.74 & 6.04 & 6.27 & 6.27 \\
\hline cdk2 & $1 \mathrm{wcc}$ & $2 \mathrm{bts}$ & 7.32 & 7.67 & 6.02 & 7.45 & 7.87 \\
\hline cdk2 & 1wcc & $2 \mathrm{c} 6 \mathrm{k}$ & 2.88 & 2.14 & 1.93 & 2.25 & - \\
\hline chk1 & $2 \mathrm{c} 3 \mathrm{j}$ & 1nvs & 1.65 & 5.15 & 1.71 & 5.14 & - \\
\hline chk1 & $2 \mathrm{c} 3 \mathrm{j}$ & $2 \mathrm{cgu}$ & 1.34 & 1.46 & 2.87 & 2.81 & 3.37 \\
\hline chk1 & $2 \mathrm{e} 9 \mathrm{o}$ & $2 \mathrm{cgu}$ & 1.24 & 2.75 & 2.88 & 2.77 & - \\
\hline chk1 & $2 \mathrm{e} 9 \mathrm{o}$ & $2 \operatorname{cg} x$ & 5.49 & 6.58 & 6.44 & 2.13 & 2.55 \\
\hline dpp4 & $1 \mathrm{n} 1 \mathrm{~m}$ & $1 \times 70$ & 2.75 & 5.12 & 3.16 & 9.71 & - \\
\hline $\mathrm{dpp} 4$ & 1rwq & $1 \times 70$ & 2.32 & 2.18 & 2.75 & 5.12 & 4.71 \\
\hline fxa & $1 \mathrm{~g} 2 \mathrm{~m}$ & $11 \mathrm{pk}$ & 7.85 & 8.21 & 3.87 & 3.87 & 3.88 \\
\hline fxa & $\operatorname{lnfx}$ & 1lpk & 10.30 & 10.00 & 10.03 & 10.00 & 3.69 \\
\hline fxa & $\operatorname{lnfx}$ & $11 \mathrm{pz}$ & 10.16 & 8.10 & 10.10 & 10.28 & - \\
\hline fxa & $\operatorname{lnfx}$ & 1lqd & 10.17 & 10.05 & 10.20 & 10.20 & 10.14 \\
\hline fxa & 1xka & 1lqd & 9.63 & 8.02 & 9.71 & 8.33 & - \\
\hline pka & $2 \mathrm{c} 1 \mathrm{a}$ & $1 \mathrm{bx} 6$ & 2.51 & 2.49 & 2.49 & 2.53 & 6.25 \\
\hline pka & $2 \mathrm{erz}$ & $1 \mathrm{cdk}$ & 2.23 & 2.36 & 4.34 & 4.47 & 4.21 \\
\hline pka & 2erz & $1 \mathrm{svg}$ & 10.27 & 10.18 & 10.22 & 10.24 & 10.25 \\
\hline ppar & 2 prg & 1zgy & 0.59 & 1.08 & 2.01 & 2.05 & - \\
\hline ppar & $2 \mathrm{prg}$ & 2ath & 4.59 & 4.56 & 5.31 & 5.47 & 5.43 \\
\hline ppar & 2 prg & $2 \mathrm{gtk}$ & 1.45 & 1.87 & 2.22 & 0.71 & 2.48 \\
\hline throm & 1ae8 & $\operatorname{lgj} 4$ & 8.88 & 8.76 & 8.86 & 8.77 & 9.59 \\
\hline throm & 1ae8 & 1gj5 & 5.95 & 5.95 & 5.99 & 8.27 & 8.27 \\
\hline throm & 1ae8 & $102 \mathrm{~g}$ & 5.98 & 9.12 & 9.11 & 8.95 & 9.11 \\
\hline throm & 1ae8 & $105 \mathrm{~g}$ & 9.50 & 9.35 & 8.58 & 8.35 & - \\
\hline throm & 1ae8 & 1riw & 8.04 & 4.93 & 4.91 & 8.47 & 9.22 \\
\hline throm & $1 \mathrm{k} 21$ & 1riw & 3.64 & 4.43 & 4.13 & 4.95 & 4.65 \\
\hline throm & 1nm6 & $1 \mathrm{fpc}$ & 5.02 & 4.76 & 4.96 & 4.61 & - \\
\hline
\end{tabular}


dependence of magnitude of the stability score on the distance between the anchor atoms and the ligand, allowed us move to a simple linear combination of the two scores. A final fit of $\mathrm{D}-5 \mathrm{H}$, where $\mathrm{D}$ is the stability score and $\mathrm{H}$ is the interaction persistence score achieved the best results.

The results for this modification of the algorithm are presented in figure(s) 1 and are similar to the results presented in the publication. Of the 42 cases run, 37 are within 2.0 angstrom heavy-atom RMSD of the native conformation and 40 are within 2.5 angstrom RMSD. There is not much correlation between the systems that this version of the algorithm had difficulties with and those that the version presented in the paper. This would suggest that there is some room for improvement in both methods as the subtle differences between the collective variables used could be the source of the failures. Combining together results generated with multiple collective variables, or further refinements to the existing collective variable, could potentially result in more accurate predictions. Even in their current form, the successful implementation of two variants of this method further demonstrates the ability of metadynamics-based methods to effectively discriminate between native ligand poses and high quality decoys without a reliance on any specific collective variable.

\section{Effect of Increasing metadynamics strength: Hill Height}

The other parameter aside from the choice of CV that can make some difference in the results of these short trial runs is the height of the metadynamics potential, $h$. We show some example cases of the effects of increasing this parameter. In many cases the separation of non-native-like poses becomes clearer with increasing hill height. In several cases unbiased MD trials and weak metadynamics fail to clearly reject non-native-like poses, while large hill heights do. In cases where non-native-like poses are well-separated in unbiased MD and weak metadynamics, increasing hill height generally leaves the poses well differentiated, except in the dpp4 cases noted in the main text, where the large RMSD estimates can be taken as 


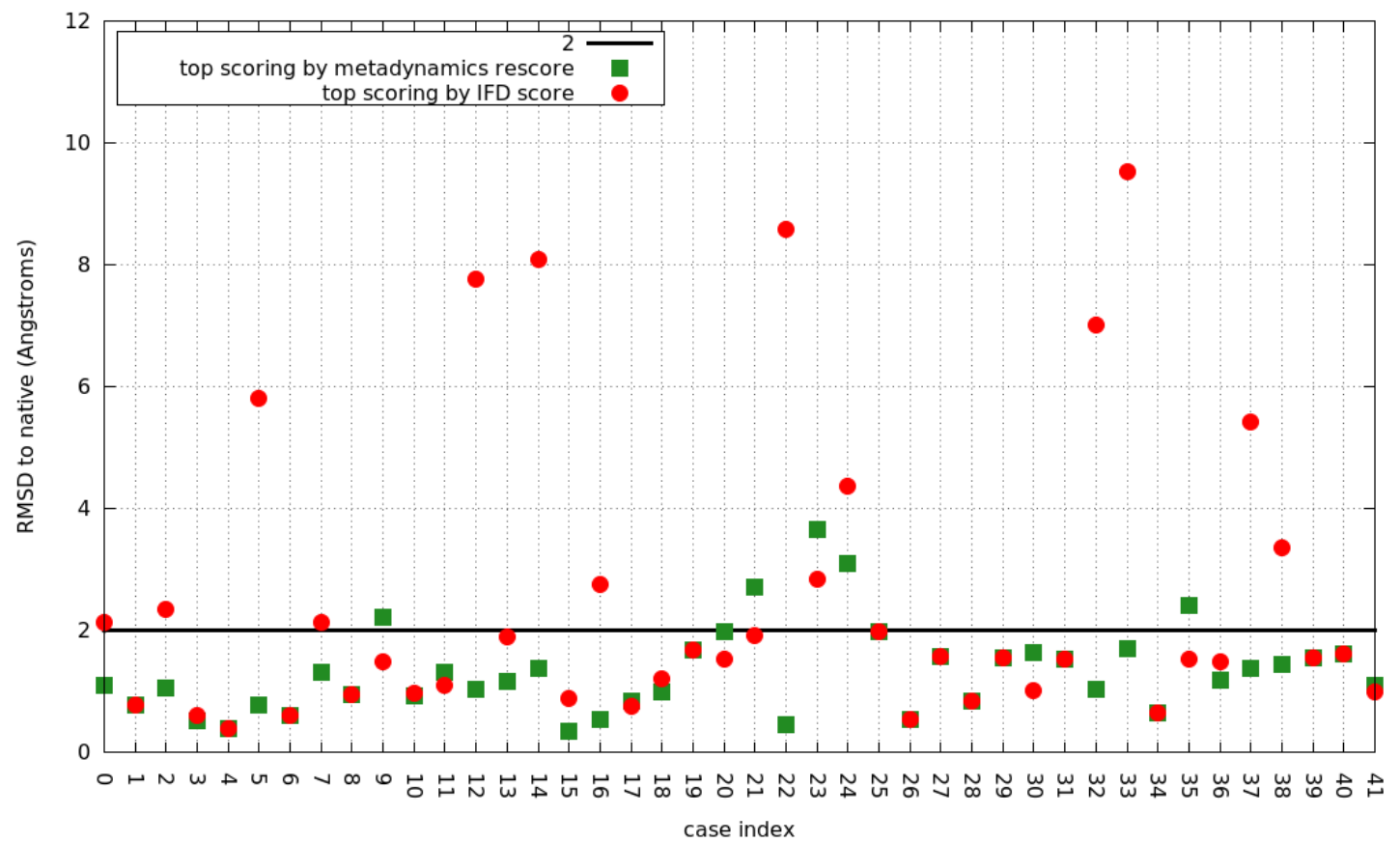

Figure 1: RMSD to native (crystallographically determined binding mode) for top-scoring pose by IFD score (red circles) shown compared to the RMSD to native of the top-scoring pose with metadynamics rescoring (green squares) shows the significant reduction in nonnative-like poses detected by metadynamics rescoring

diagnostic to require reduction of the hill height. We therefore take the default hill height in the anchor-RMSD protocol to be $0.3 \mathrm{kcal} / \mathrm{mol}$, which is approximately half of the characteristic thermal energy of the system, $k_{B} T$. For comparison of unbiased MD simulations, a simple running average of the CV value is used in place of the RMSD estimate, as there is no bias potential generated from which to estimate the free energy. Figure 2 shows an example of ramping up from unbiased MD through weak and strong hill height metadynamics where a clearly non-native-like structure would be strongly preferred by the RMSD estimates from unbiased MD and weak metadynamics, but are rejected by the strong metadynamics. Figure 3 shows another example where a moderately non-native-like structure is increasingly separated with increasing hill height (in this case the competing structures are nearly tied, but hydrogen bond persistence would clearly choose the sub-2.0 pose). 

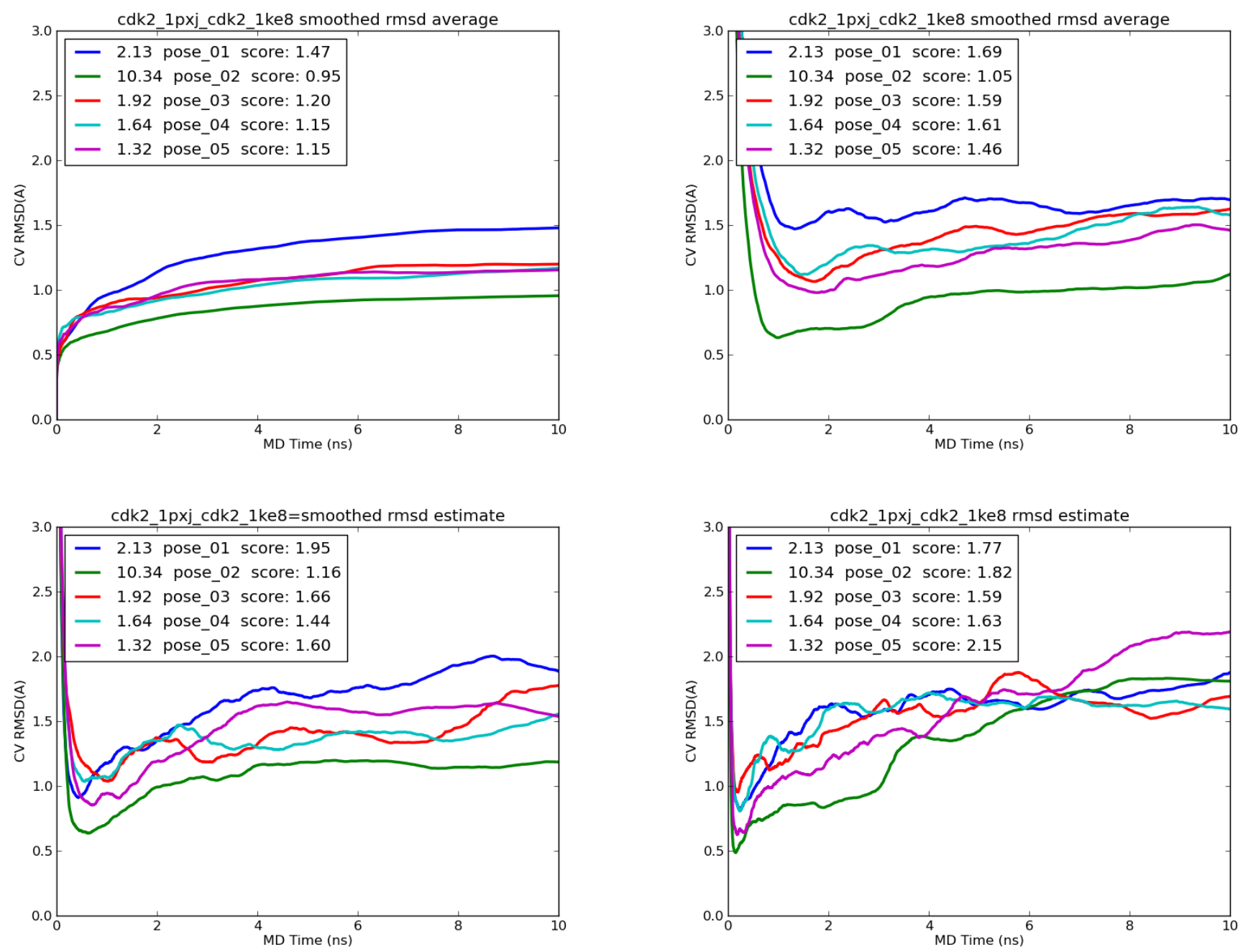

Figure 2: Plots of the rmsd estimate obtained with unbiased Molecular dynamics (top left), weak metadynamics (top right - hill height $0.05 \mathrm{kcal} / \mathrm{mol}$ ), intermediate metadynamics (bottom left - hill height $0.125 \mathrm{kcal} / \mathrm{mol}$ ), and strong metadynamics (bottom right - hill height $0.3 \mathrm{kcal} / \mathrm{mol}$ ) for the case of the ligand from PDB structure $1 \mathrm{ke} 8$ docked with the cdk2 receptor from $\mathrm{PDB}$ 1pxj. For unbiased MD, a running average of the $\mathrm{CV}$ value is used in place of the the rmsd estimate. 

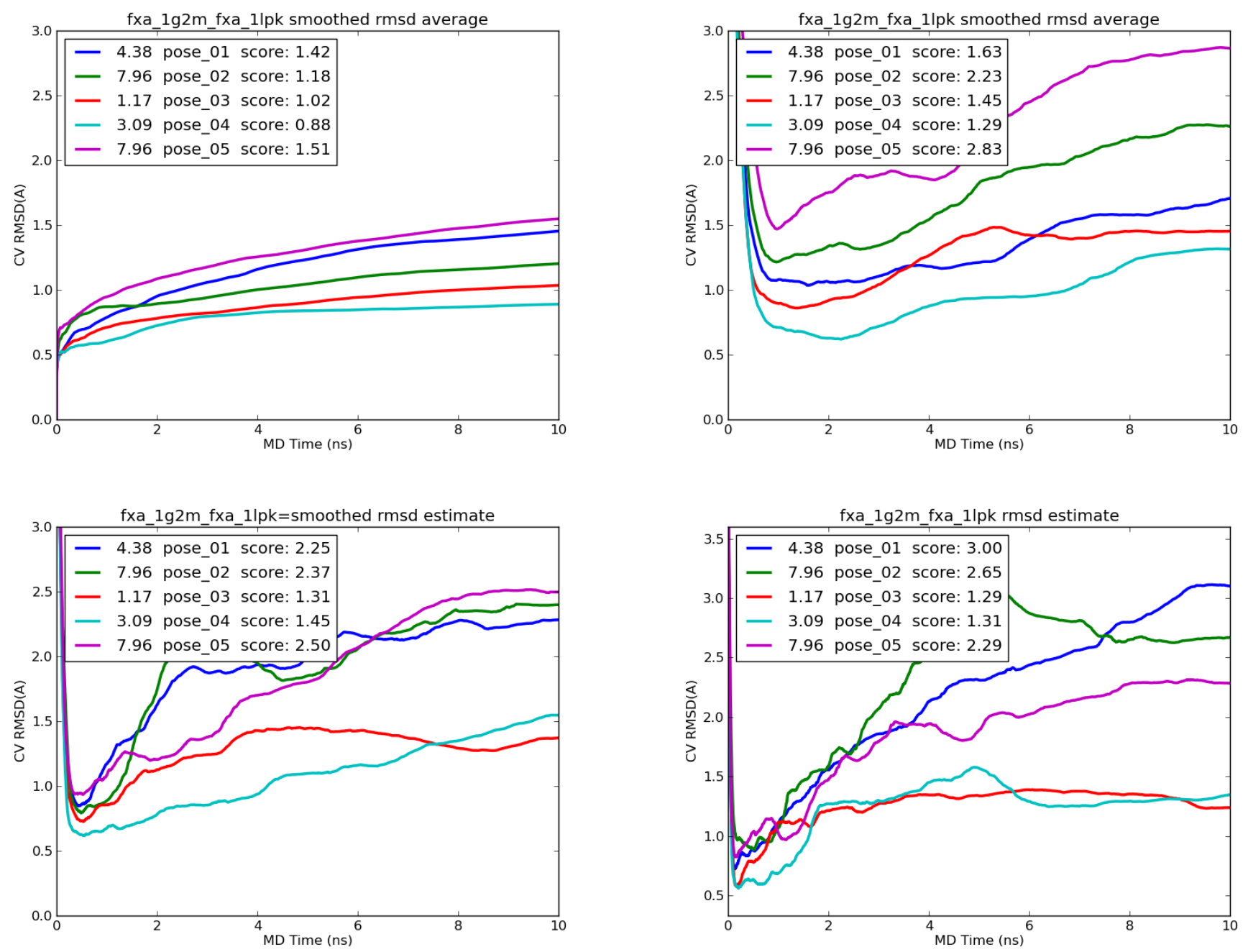

Figure 3: Plots of the rmsd estimate obtained with unbiased Molecular dynamics (top left), weak metadynamics (top right - hill height $0.05 \mathrm{kcal} / \mathrm{mol}$ ), intermediate metadynamics (bottom left - hill height $0.125 \mathrm{kcal} / \mathrm{mol}$ ), and strong metadynamics (bottom right - hill height $0.3 \mathrm{kcal} / \mathrm{mol}$ ) for the case of the ligand from PDB structure 1lpk docked with the fxa receptor from PDB $1 \mathrm{~g} 2 \mathrm{~m}$. For unbiased MD, a running average of the CV value is used in place of the the rmsd estimate. 


\section{Full results}

In table 2, the results of each case ranked by combined score are shown along with the rmsd to native, with the rmsd to native of the best scoring pose in bold face. In addition, the anchor atoms used for each case are listed under the receptor PDB id for each case is specified by chain name and residue number (only the backbone heavy atoms of the specified residues were included in the anchor region). The same anchor atoms were used for all 5 poses in a given case.

\begin{tabular}{|c|c|c|c|c|c|c|c|c|}
\hline Case & receptor & ligand & & rank 1 & rank 2 & rank 3 & rank 4 & rank 5 \\
\hline \multirow{2}{*}{ 1. alr2 } & 1iei & $2 \mathrm{fz} 8$ & combined score: & 2.29 & 2.15 & 1.82 & 1.42 & 1.19 \\
\hline & A:30-33 & & RMSD to native: & 0.53 & 1.10 & 1.46 & 2.36 & 2.14 \\
\hline \multirow[t]{2}{*}{ 2. alr2 } & $2 \mathrm{fzd}$ & 1iei & combined score: & 2.37 & 1.51 & 1.05 & 0.98 & 0.56 \\
\hline & $A: 54-57$ & & RMSD to native: & 0.78 & 7.06 & 1.88 & 1.26 & 3.37 \\
\hline \multirow[t]{2}{*}{ 3. alr2 } & $2 \mathrm{fzd}$ & $1 \mathrm{t} 40$ & combined score: & 2.20 & 1.30 & 1.12 & 1.09 & 0.94 \\
\hline & A:54-57 & & RMSD to native: & 1.05 & 2.35 & 2.47 & 10.50 & 5.32 \\
\hline \multirow[t]{2}{*}{ 4. alr2 } & $2 \mathrm{fzd}$ & $1 z 3 n$ & combined score: & 2.87 & 2.43 & 2.37 & 2.28 & 1.35 \\
\hline & A:54-57 & & RMSD to native: & 0.53 & 0.60 & 0.68 & 0.69 & 2.33 \\
\hline \multirow[t]{2}{*}{ 5. alr2 } & $2 \mathrm{fzd}$ & $2 \mathrm{hvn}$ & combined score: & 2.47 & 2.28 & 2.27 & 2.12 & 0.75 \\
\hline & A:30-33 & & RMSD to native: & 1.36 & 0.40 & 9.50 & 5.77 & 9.87 \\
\hline \multirow[t]{2}{*}{ 6. alr2 } & $2 \mathrm{fzd}$ & $2 \mathrm{ikg}$ & combined score: & 1.85 & 1.09 & 0.77 & 0.60 & 0.38 \\
\hline & A:88-91 & & RMSD to native: & 0.79 & 4.75 & 6.03 & 5.22 & 5.81 \\
\hline \multirow[t]{2}{*}{ 7. $\operatorname{cdk} 2$} & $1 \mathrm{pxj}$ & 1jvp & combined score: & 1.96 & 1.64 & 0.73 & 0.56 & 0.00 \\
\hline & $\mathrm{A}: 211-214$ & & RMSD to native: & 0.61 & 0.75 & 6.32 & 6.42 & 5.28 \\
\hline \multirow[t]{2}{*}{ 8. $\operatorname{cdk} 2$} & $1 \mathrm{pxj}$ & 1ke8 & combined score: & 1.79 & 1.61 & 1.19 & 1.00 & 0.13 \\
\hline & $\mathrm{A}: 211-214$ & & RMSD to native: & 1.92 & 1.64 & 1.32 & 2.13 & 10.34 \\
\hline \multirow[t]{2}{*}{ 9. $\operatorname{cdk} 2$} & $1 \mathrm{pxj}$ & $1 \mathrm{pxo}$ & combined score: & 2.52 & 2.20 & 1.86 & 1.57 & 0.00 \\
\hline & $\mathrm{A}: 211-214$ & & RMSD to native: & 0.96 & 1.04 & 1.63 & 2.83 & 2.48 \\
\hline
\end{tabular}




\begin{tabular}{|c|c|c|c|c|c|c|c|c|}
\hline 10. cdk2 & $\begin{array}{l}1 \mathrm{pxj} \\
\mathrm{A}: 211-214\end{array}$ & $2 \mathrm{a} 0 \mathrm{c}$ & $\begin{array}{l}\text { combined score: } \\
\text { RMSD to native: }\end{array}$ & $\begin{array}{l}1.82 \\
\mathbf{0 . 8 3}\end{array}$ & $\begin{array}{l}1.75 \\
2.21\end{array}$ & $\begin{array}{l}1.64 \\
0.90\end{array}$ & $\begin{array}{l}1.59 \\
1.49\end{array}$ & $\begin{array}{l}1.07 \\
1.61\end{array}$ \\
\hline 11. cdk2 & $\begin{array}{l}1 \mathrm{pxj} \\
\mathrm{A}: 211-214\end{array}$ & $2 \mathrm{btr}$ & $\begin{array}{l}\text { combined score: } \\
\text { RMSD to native: }\end{array}$ & $\begin{array}{l}2.67 \\
\mathbf{0 . 9 4}\end{array}$ & $\begin{array}{l}2.03 \\
0.98\end{array}$ & $\begin{array}{l}1.96 \\
0.50\end{array}$ & $\begin{array}{l}1.80 \\
1.44\end{array}$ & $\begin{array}{l}0.02 \\
7.88\end{array}$ \\
\hline 12. cdk2 & $\begin{array}{l}1 \mathrm{pxj} \\
\mathrm{A}: 211-214\end{array}$ & $2 \mathrm{bts}$ & $\begin{array}{l}\text { combined score: } \\
\text { RMSD to native: }\end{array}$ & $\begin{array}{l}2.11 \\
\mathbf{1 . 3 1}\end{array}$ & $\begin{array}{l}1.70 \\
1.10\end{array}$ & $\begin{array}{l}0.51 \\
8.15\end{array}$ & $\begin{array}{l}0.04 \\
2.13\end{array}$ & $\begin{array}{l}0.00 \\
7.60\end{array}$ \\
\hline 13. cdk2 & $\begin{array}{l}1 \mathrm{pxj} \\
\mathrm{A}: 211-214\end{array}$ & $2 \mathrm{c} 6 \mathrm{~m}$ & $\begin{array}{l}\text { combined score: } \\
\text { RMSD to native: }\end{array}$ & $\begin{array}{l}1.33 \\
\mathbf{1 . 0 4}\end{array}$ & $\begin{array}{l}0.56 \\
8.69\end{array}$ & $\begin{array}{l}0.51 \\
7.77\end{array}$ & $\begin{array}{l}0.05 \\
8.72\end{array}$ & $\begin{array}{l}0.00 \\
4.93\end{array}$ \\
\hline 14. cdk2 & $\begin{array}{l}1 \mathrm{wcc} \\
\text { A:211-214 }\end{array}$ & 1oit & $\begin{array}{l}\text { combined score: } \\
\text { RMSD to native: }\end{array}$ & $\begin{array}{l}1.90 \\
\mathbf{1 . 9 0}\end{array}$ & $\begin{array}{l}1.73 \\
1.16\end{array}$ & $\begin{array}{l}1.32 \\
6.71\end{array}$ & $\begin{array}{l}1.20 \\
6.87\end{array}$ & $\begin{array}{l}0.00 \\
6.57\end{array}$ \\
\hline 15. cdk2 & $\begin{array}{l}1 \mathrm{wcc} \\
\mathrm{A}: 17-20\end{array}$ & $2 \mathrm{~b} 52$ & $\begin{array}{l}\text { combined score: } \\
\text { RMSD to native: }\end{array}$ & $\begin{array}{l}1.39 \\
\mathbf{6 . 2 4}\end{array}$ & $\begin{array}{l}0.86 \\
1.39\end{array}$ & $\begin{array}{l}0.78 \\
1.85\end{array}$ & $\begin{array}{l}0.60 \\
8.08\end{array}$ & $\begin{array}{l}0.06 \\
7.96\end{array}$ \\
\hline 16. cdk2 & $\begin{array}{l}1 \mathrm{wcc} \\
\text { A:211-214 }\end{array}$ & 2bhe & $\begin{array}{l}\text { combined score: } \\
\text { RMSD to native: }\end{array}$ & $\begin{array}{l}2.01 \\
\mathbf{1 . 1 8}\end{array}$ & $\begin{array}{l}1.62 \\
0.36\end{array}$ & $\begin{array}{l}0.86 \\
4.49\end{array}$ & $\begin{array}{l}0.37 \\
6.28\end{array}$ & $\begin{array}{l}0.05 \\
0.88\end{array}$ \\
\hline 17. cdk2 & $\begin{array}{l}1 \mathrm{wcc} \\
\text { A:211-214 }\end{array}$ & $2 \mathrm{bts}$ & $\begin{array}{l}\text { combined score: } \\
\text { RMSD to native: }\end{array}$ & $\begin{array}{l}2.40 \\
2.77\end{array}$ & $\begin{array}{l}1.78 \\
0.69\end{array}$ & $\begin{array}{l}1.70 \\
1.07\end{array}$ & $\begin{array}{l}1.54 \\
0.54\end{array}$ & $\begin{array}{l}1.51 \\
2.54\end{array}$ \\
\hline 18. cdk2 & $\begin{array}{l}1 \mathrm{wcc} \\
\text { A:211-214 }\end{array}$ & $2 \mathrm{c} 6 \mathrm{k}$ & $\begin{array}{l}\text { combined score: } \\
\text { RMSD to native: }\end{array}$ & $\begin{array}{l}1.51 \\
\mathbf{0 . 8 5}\end{array}$ & $\begin{array}{l}1.50 \\
0.75\end{array}$ & $\begin{array}{l}0.98 \\
1.63\end{array}$ & $\begin{array}{l}0.73 \\
1.20\end{array}$ & $\begin{array}{l}0.13 \\
6.99\end{array}$ \\
\hline 19. $\operatorname{chk} 1$ & $\begin{array}{l}2 \mathrm{c} 3 \mathrm{j} \\
\mathrm{A}: 239-242\end{array}$ & 1nvs & $\begin{array}{l}\text { combined score: } \\
\text { RMSD to native: }\end{array}$ & $\begin{array}{l}3.71 \\
\mathbf{1 . 0 1}\end{array}$ & $\begin{array}{l}3.49 \\
1.22\end{array}$ & $\begin{array}{l}0.33 \\
5.04\end{array}$ & $\begin{array}{l}0.00 \\
4.66\end{array}$ & $\begin{array}{l}0.00 \\
4.82\end{array}$ \\
\hline 20. $\operatorname{chk} 1$ & $\begin{array}{l}2 \mathrm{c} 3 \mathrm{j} \\
\mathrm{A}: 239-242\end{array}$ & $2 \operatorname{cgu}$ & $\begin{array}{l}\text { combined score: } \\
\text { RMSD to native: }\end{array}$ & $\begin{array}{l}2.21 \\
\mathbf{2 . 1 4}\end{array}$ & $\begin{array}{l}2.08 \\
1.68\end{array}$ & $\begin{array}{l}2.08 \\
2.25\end{array}$ & $\begin{array}{l}1.58 \\
3.03\end{array}$ & $\begin{array}{l}0.83 \\
3.98\end{array}$ \\
\hline 21. $\operatorname{chk} 1$ & $\begin{array}{l}2 \mathrm{e} 9 \mathrm{o} \\
\mathrm{A}: 239-242\end{array}$ & $2 \operatorname{cgu}$ & $\begin{array}{l}\text { combined score: } \\
\text { RMSD to native: }\end{array}$ & $\begin{array}{l}2.06 \\
\mathbf{1 . 9 8}\end{array}$ & $\begin{array}{l}2.00 \\
2.23\end{array}$ & $\begin{array}{l}1.95 \\
1.54\end{array}$ & $\begin{array}{l}1.52 \\
2.69\end{array}$ & $\begin{array}{l}0.92 \\
2.35\end{array}$ \\
\hline 22. $\operatorname{chk} 1$ & $\begin{array}{l}2 \mathrm{e} 9 \mathrm{o} \\
\mathrm{A}: 239-242\end{array}$ & $2 \operatorname{cg} x$ & $\begin{array}{l}\text { combined score: } \\
\text { RMSD to native: }\end{array}$ & $\begin{array}{l}0.75 \\
\mathbf{1 . 6 6}\end{array}$ & $\begin{array}{l}0.30 \\
1.93\end{array}$ & $\begin{array}{l}0.17 \\
2.92\end{array}$ & $\begin{array}{l}0.13 \\
2.03\end{array}$ & $\begin{array}{l}0.07 \\
2.71\end{array}$ \\
\hline
\end{tabular}




\begin{tabular}{|c|c|c|c|c|c|c|c|c|}
\hline $\begin{array}{l}\text { 23. dpp4 } \\
(h=0.05)\end{array}$ & $\begin{array}{l}\text { 1n1m } \\
\text { A:748-751 }\end{array}$ & $1 \times 70$ & $\begin{array}{l}\text { combined score: } \\
\text { RMSD to native: }\end{array}$ & $\begin{array}{l}0.77 \\
\mathbf{0 . 4 6}\end{array}$ & $\begin{array}{l}0.28 \\
6.64\end{array}$ & $\begin{array}{l}0.02 \\
3.19\end{array}$ & $\begin{array}{l}0.00 \\
8.59\end{array}$ & $\begin{array}{l}0.00 \\
3.54\end{array}$ \\
\hline $\begin{array}{l}\text { 24. dpp4 } \\
(h=0.05)\end{array}$ & $\begin{array}{l}\text { 1rwq } \\
\text { A:748-751 }\end{array}$ & $1 \times 70$ & $\begin{array}{l}\text { combined score: } \\
\text { RMSD to native: }\end{array}$ & $\begin{array}{l}0.10 \\
\mathbf{1 . 9 0}\end{array}$ & $\begin{array}{l}0.04 \\
4.61\end{array}$ & $\begin{array}{l}0.00 \\
3.66\end{array}$ & $\begin{array}{l}0.00 \\
2.52\end{array}$ & $\begin{array}{l}0.00 \\
2.84\end{array}$ \\
\hline 25. fxa & $\begin{array}{l}1 \mathrm{~g} 2 \mathrm{~m} \\
\mathrm{~A}: 31-34\end{array}$ & 1lpk & $\begin{array}{l}\text { combined score: } \\
\text { RMSD to native: }\end{array}$ & $\begin{array}{l}1.86 \\
\mathbf{1 . 1 7}\end{array}$ & $\begin{array}{l}1.08 \\
7.96\end{array}$ & $\begin{array}{l}1.06 \\
7.96\end{array}$ & $\begin{array}{l}0.94 \\
4.38\end{array}$ & $\begin{array}{l}0.90 \\
3.09\end{array}$ \\
\hline 26. fxa & $\begin{array}{l}\operatorname{lnfx} \\
A: 31-34\end{array}$ & 1lpk & $\begin{array}{l}\text { combined score: } \\
\text { RMSD to native: }\end{array}$ & $\begin{array}{l}2.07 \\
\mathbf{1 . 9 8}\end{array}$ & $\begin{array}{l}1.96 \\
0.79\end{array}$ & $\begin{array}{l}1.92 \\
9.04\end{array}$ & $\begin{array}{l}1.54 \\
7.64\end{array}$ & $\begin{array}{l}1.06 \\
7.80\end{array}$ \\
\hline 27. fxa & $\begin{array}{l}\operatorname{lnfx} \\
\text { A:105-108 }\end{array}$ & $1 \mathrm{lpz}$ & $\begin{array}{l}\text { combined score: } \\
\text { RMSD to native: }\end{array}$ & $\begin{array}{l}2.52 \\
\mathbf{0 . 5 4}\end{array}$ & $\begin{array}{l}1.85 \\
6.58\end{array}$ & $\begin{array}{l}1.60 \\
0.82\end{array}$ & $\begin{array}{l}1.22 \\
6.31\end{array}$ & $\begin{array}{l}0.01 \\
9.70\end{array}$ \\
\hline 28. fxa & $\begin{array}{l}\operatorname{lnfx} \\
\text { C:105-108 }\end{array}$ & 1lqd & $\begin{array}{l}\text { combined score: } \\
\text { RMSD to native: }\end{array}$ & $\begin{array}{l}2.24 \\
\mathbf{1 . 1 4}\end{array}$ & $\begin{array}{l}1.90 \\
1.58\end{array}$ & $\begin{array}{l}1.57 \\
4.54\end{array}$ & $\begin{array}{l}1.54 \\
3.75\end{array}$ & $\begin{array}{l}1.08 \\
6.17\end{array}$ \\
\hline 29. fxa & $\begin{array}{l}\text { 1xka } \\
\text { C:31-34 }\end{array}$ & 1lqd & $\begin{array}{l}\text { combined score: } \\
\text { RMSD to native: }\end{array}$ & $\begin{array}{l}2.21 \\
\mathbf{0 . 8 4}\end{array}$ & $\begin{array}{l}1.50 \\
4.42\end{array}$ & $\begin{array}{l}1.16 \\
6.74\end{array}$ & $\begin{array}{l}0.98 \\
6.74\end{array}$ & $\begin{array}{l}0.00 \\
7.51\end{array}$ \\
\hline 30. pka & $\begin{array}{l}2 \mathrm{c} 1 \mathrm{a} \\
\mathrm{A}: 222-225\end{array}$ & $1 b x 6$ & $\begin{array}{l}\text { combined score: } \\
\text { RMSD to native: }\end{array}$ & $\begin{array}{l}1.46 \\
\mathbf{1 . 5 6}\end{array}$ & $\begin{array}{l}1.02 \\
2.64\end{array}$ & $\begin{array}{l}0.66 \\
5.81\end{array}$ & $\begin{array}{l}0.14 \\
6.00\end{array}$ & $\begin{array}{l}0.03 \\
6.37\end{array}$ \\
\hline 31. pka & $\begin{array}{l}2 \mathrm{erz} \\
\text { E:81-89 }\end{array}$ & $1 \mathrm{cdk}$ & $\begin{array}{l}\text { combined score: } \\
\text { RMSD to native: }\end{array}$ & $\begin{array}{l}0.06 \\
\mathbf{1 . 6 3}\end{array}$ & $\begin{array}{l}0.00 \\
2.69\end{array}$ & $\begin{array}{l}0.00 \\
2.31\end{array}$ & $\begin{array}{l}0.00 \\
1.02\end{array}$ & $\begin{array}{l}0.00 \\
1.89\end{array}$ \\
\hline 32. pka & $\begin{array}{l}2 \mathrm{erz} \\
\text { E:222-225 }\end{array}$ & $1 \mathrm{svg}$ & $\begin{array}{l}\text { combined score: } \\
\text { RMSD to native: }\end{array}$ & $\begin{array}{l}1.84 \\
\mathbf{1 . 5 3}\end{array}$ & $\begin{array}{l}0.44 \\
3.36\end{array}$ & $\begin{array}{l}0.23 \\
10.30\end{array}$ & $\begin{array}{l}0.06 \\
4.42\end{array}$ & $\begin{array}{l}0.02 \\
9.86\end{array}$ \\
\hline 33. ppar & $\begin{array}{l}2 \text { prg } \\
\text { A:255-258 }\end{array}$ & 1zgy & $\begin{array}{l}\text { combined score: } \\
\text { RMSD to native: }\end{array}$ & $\begin{array}{l}1.54 \\
\mathbf{1 . 0 4}\end{array}$ & $\begin{array}{l}0.72 \\
5.93\end{array}$ & $\begin{array}{l}0.39 \\
6.73\end{array}$ & $\begin{array}{l}0.02 \\
7.08\end{array}$ & $\begin{array}{l}0.01 \\
7.02\end{array}$ \\
\hline 34. ppar & $\begin{array}{l}2 \mathrm{prg} \\
\text { A:255-258 }\end{array}$ & 2ath & $\begin{array}{l}\text { combined score: } \\
\text { RMSD to native: }\end{array}$ & $\begin{array}{l}1.35 \\
\mathbf{1 . 7 1}\end{array}$ & $\begin{array}{l}0.08 \\
4.83\end{array}$ & $\begin{array}{l}0.06 \\
9.40\end{array}$ & $\begin{array}{l}0.00 \\
3.14\end{array}$ & $\begin{array}{l}0.00 \\
9.53\end{array}$ \\
\hline 35. ppar & $\begin{array}{l}2 \text { prg } \\
\text { A:233-236 }\end{array}$ & $2 \mathrm{gtk}$ & $\begin{array}{l}\text { combined score: } \\
\text { RMSD to native: }\end{array}$ & $\begin{array}{l}1.44 \\
\mathbf{0 . 6 5}\end{array}$ & $\begin{array}{l}1.39 \\
0.96\end{array}$ & $\begin{array}{l}1.16 \\
2.27\end{array}$ & $\begin{array}{l}0.33 \\
2.07\end{array}$ & $\begin{array}{l}0.13 \\
2.12\end{array}$ \\
\hline
\end{tabular}




\begin{tabular}{|c|c|c|c|c|c|c|c|c|}
\hline 36. throm & $\begin{array}{l}\text { 1ae8 } \\
\mathrm{H}: 31-34\end{array}$ & 1riw & $\begin{array}{l}\text { combined score: } \\
\text { RMSD to native: }\end{array}$ & $\begin{array}{l}2.44 \\
2.41\end{array}$ & $\begin{array}{l}1.14 \\
6.67\end{array}$ & $\begin{array}{l}1.11 \\
1.54\end{array}$ & $\begin{array}{l}0.08 \\
8.96\end{array}$ & $\begin{array}{l}0.00 \\
9.30\end{array}$ \\
\hline 37. throm & $\begin{array}{l}1 \mathrm{k} 21 \\
\mathrm{H}: 31-34\end{array}$ & 1riw & $\begin{array}{l}\text { combined score: } \\
\text { RMSD to native: }\end{array}$ & $\begin{array}{l}1.61 \\
\mathbf{3 . 0 2}\end{array}$ & $\begin{array}{l}1.39 \\
1.19\end{array}$ & $\begin{array}{l}1.29 \\
2.09\end{array}$ & $\begin{array}{l}0.84 \\
1.48\end{array}$ & $\begin{array}{l}0.83 \\
6.73\end{array}$ \\
\hline 38. throm & $\begin{array}{l}\text { 1nm6 } \\
\text { A:31-34 }\end{array}$ & $1 \mathrm{fpc}$ & $\begin{array}{l}\text { combined score: } \\
\text { RMSD to native: }\end{array}$ & $\begin{array}{l}1.66 \\
\mathbf{1 . 2 8}\end{array}$ & $\begin{array}{l}0.88 \\
1.22\end{array}$ & $\begin{array}{l}0.76 \\
1.68\end{array}$ & $\begin{array}{l}0.62 \\
1.38\end{array}$ & $\begin{array}{l}0.00 \\
5.43\end{array}$ \\
\hline 39. throm & $\begin{array}{l}\text { 1ae8 } \\
\mathrm{H}: 31-34\end{array}$ & 1gj4 & $\begin{array}{l}\text { combined score: } \\
\text { RMSD to native: }\end{array}$ & $\begin{array}{l}3.67 \\
\mathbf{1 . 4 9}\end{array}$ & $\begin{array}{l}3.55 \\
1.61\end{array}$ & $\begin{array}{l}3.18 \\
2.11\end{array}$ & $\begin{array}{l}0.93 \\
3.39\end{array}$ & $\begin{array}{l}0.00 \\
6.69\end{array}$ \\
\hline 40. throm & $\begin{array}{l}\text { 1ae8 } \\
\mathrm{H}: 31-34\end{array}$ & 1gj5 & $\begin{array}{l}\text { combined score: } \\
\text { RMSD to native: }\end{array}$ & $\begin{array}{l}3.44 \\
0.78\end{array}$ & $\begin{array}{l}2.32 \\
6.17\end{array}$ & $\begin{array}{l}1.98 \\
6.14\end{array}$ & $\begin{array}{l}1.23 \\
2.39\end{array}$ & $\begin{array}{l}1.12 \\
2.23\end{array}$ \\
\hline 41. throm & $\begin{array}{l}1 \mathrm{ae} 8 \\
\mathrm{H}: 31-34\end{array}$ & $102 \mathrm{~g}$ & $\begin{array}{l}\text { combined score: } \\
\text { RMSD to native: }\end{array}$ & $\begin{array}{l}3.54 \\
\mathbf{1 . 6 7}\end{array}$ & $\begin{array}{l}3.24 \\
1.35\end{array}$ & $\begin{array}{l}2.86 \\
0.69\end{array}$ & $\begin{array}{l}1.92 \\
5.59\end{array}$ & $\begin{array}{l}1.56 \\
3.55\end{array}$ \\
\hline 42. throm & $\begin{array}{l}\text { 1ae8 } \\
\text { H:180-183 }\end{array}$ & $105 \mathrm{~g}$ & $\begin{array}{l}\text { combined score: } \\
\text { RMSD to native: }\end{array}$ & $\begin{array}{l}3.12 \\
\mathbf{1 . 0 9}\end{array}$ & $\begin{array}{l}2.76 \\
0.99\end{array}$ & $\begin{array}{l}2.47 \\
2.15\end{array}$ & $\begin{array}{l}1.65 \\
7.32\end{array}$ & $\begin{array}{l}0.24 \\
7.56\end{array}$ \\
\hline
\end{tabular}




\section{Convergence of HBP fraction with number of trials}

Since the HBP fraction is a raw average over trajectory frames and not an ensemble average as for the RMSD estimate, the HBP trajectories presented above are subject to a fair amount of noise. We therefore examine the convergence properties as more trials are added to the average. In most cases 10 trials appears to yield a reasonable convergence of the estimate. Below in Fig. 4, convergence plots for the three cases outlined in the main body of the text are shown, using varying number of trials to compute the HBP.

\section{HBP and RMSD estimate plots for all cases}



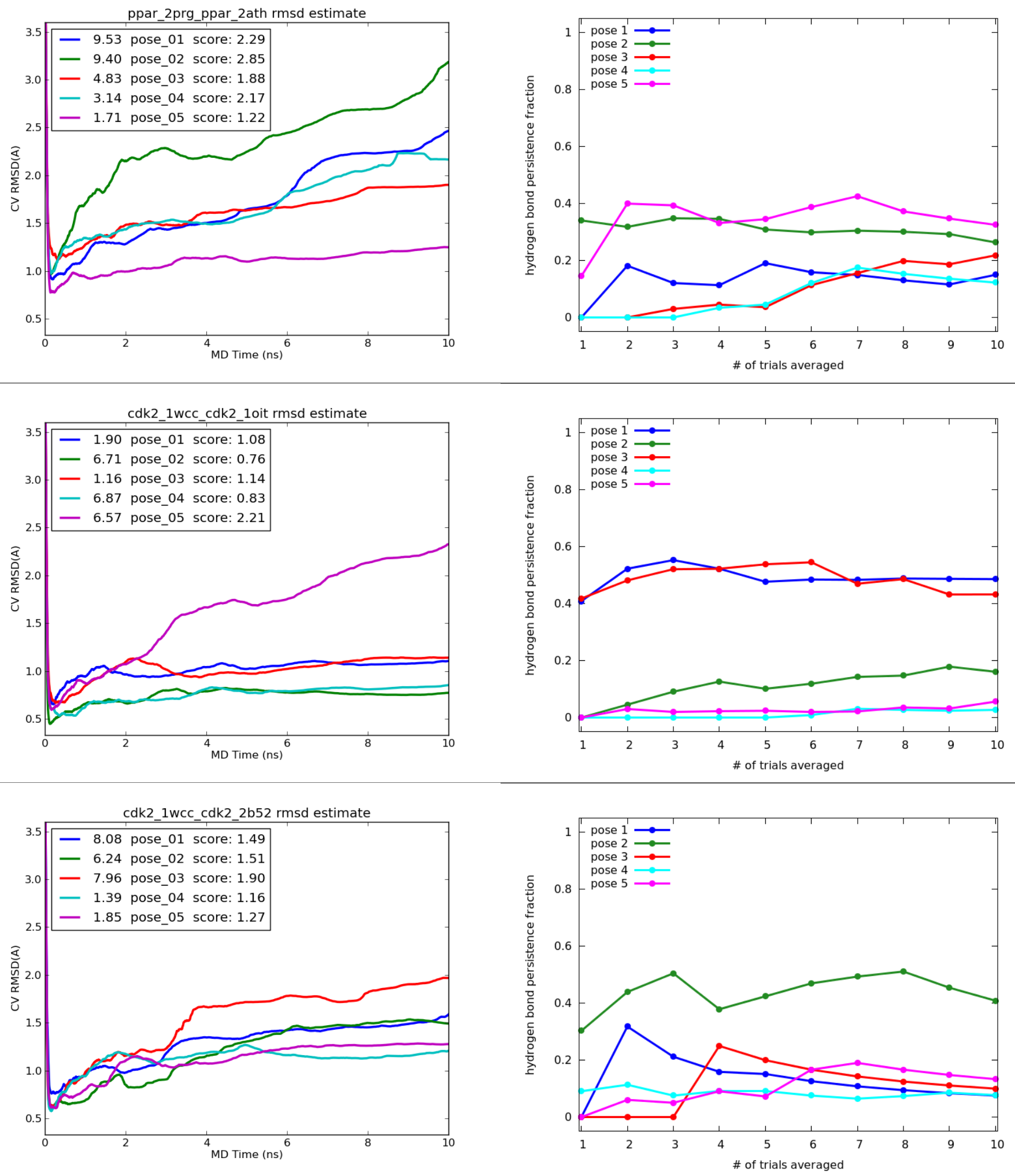

Figure 4: Plots of the averaged hydrogen bond persistence trajectories (left) and the running average of over the last $2 \mathrm{~ns}$ of the trajectories as increasing numbers of trials are included (right) for the 3 example cases highlighted in the main text. 
alr2 1iei alr2 $2 \mathrm{fz} 8 \mathrm{rmsd}$ estimate

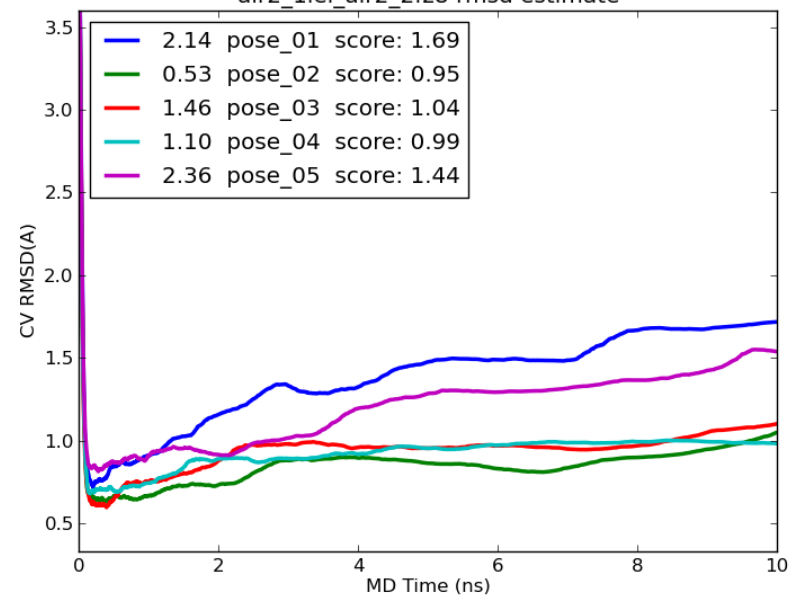

case 1

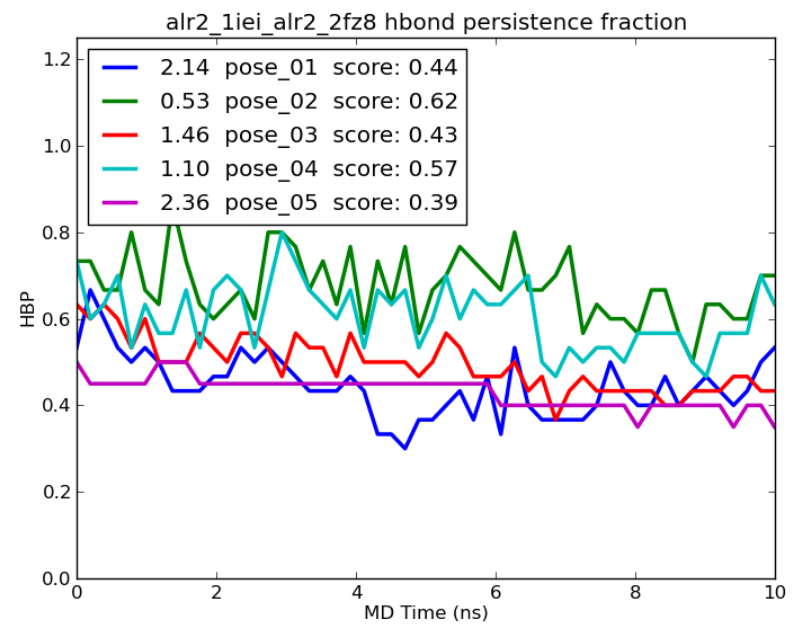

alr2 2fzd alr2 liei rmsd estimate

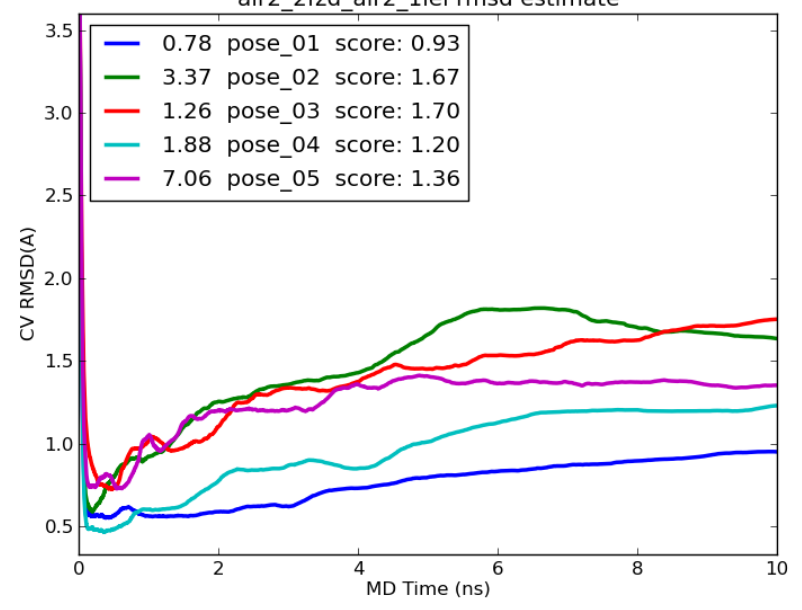

case 2
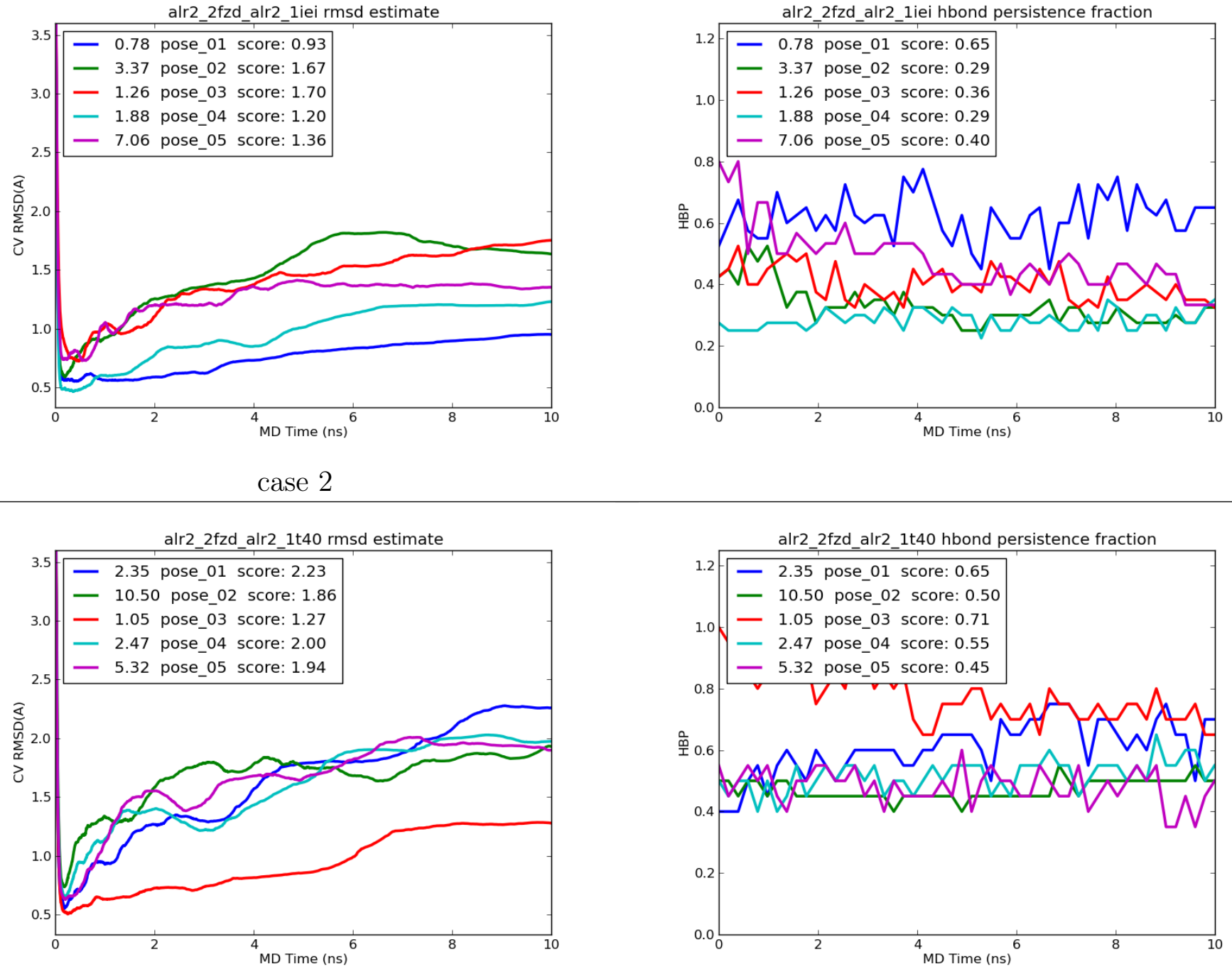

case 3 
alr2_2fzd_alr2_1z3n rmsd estimate

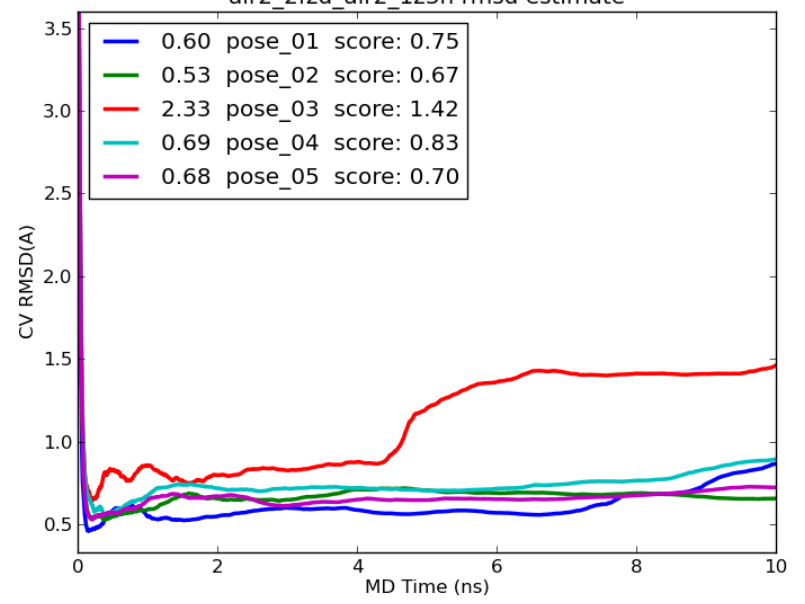

case 4
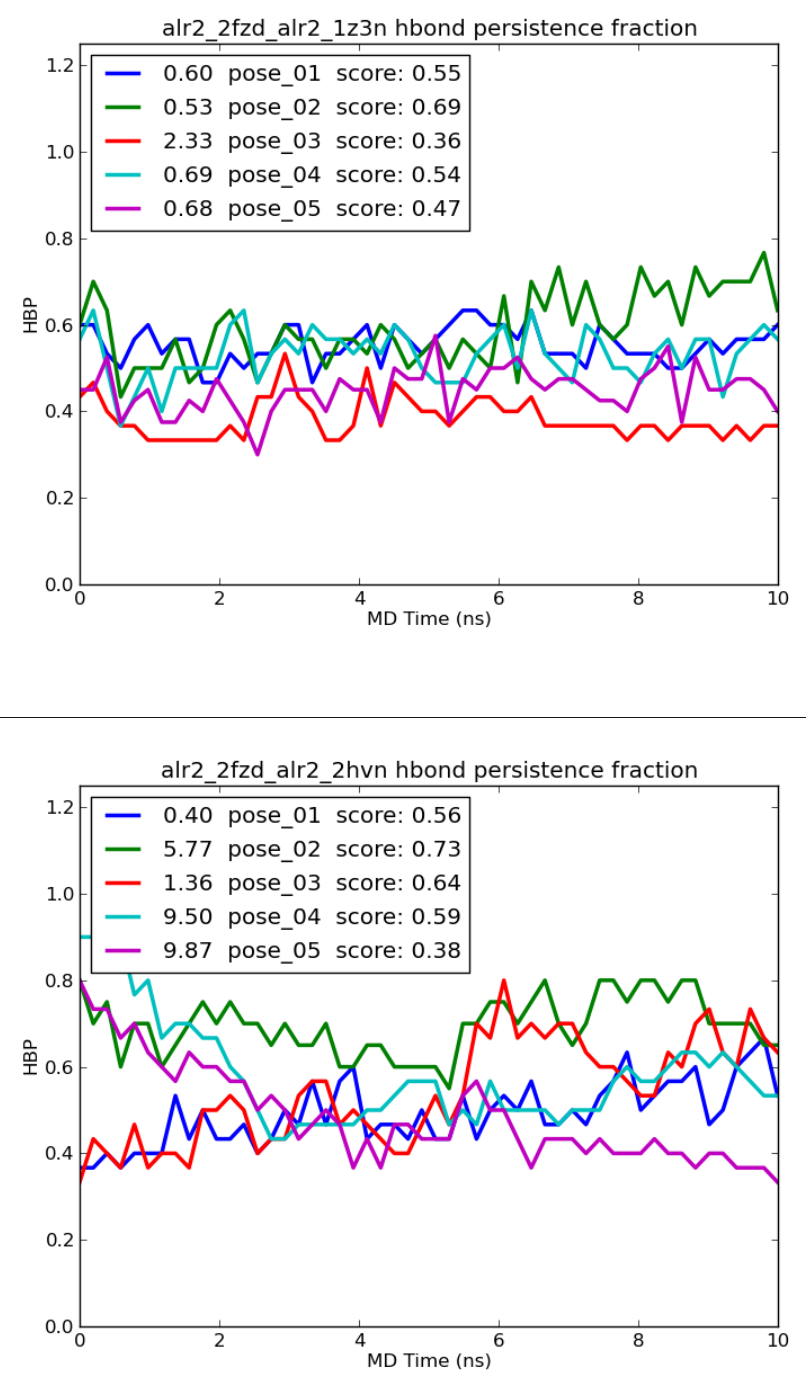

case 5
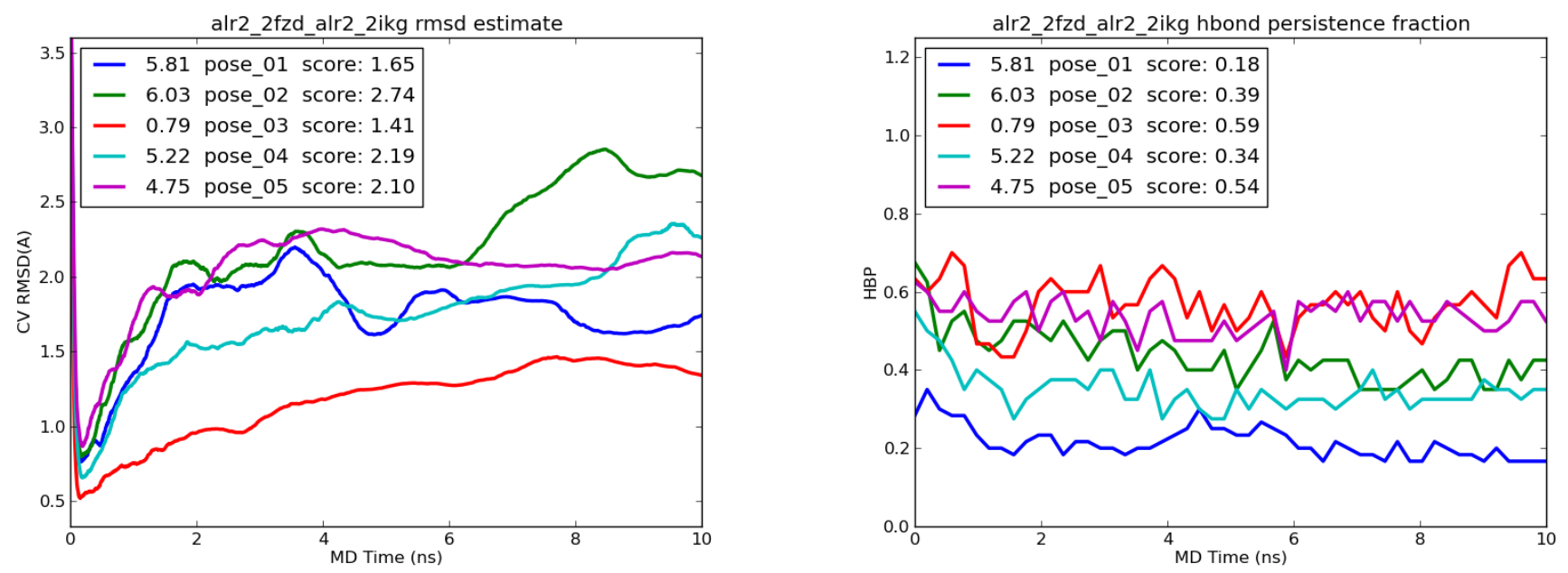

case 6 
cdk2_1pxj_cdk2_1jvp rmsd estimate

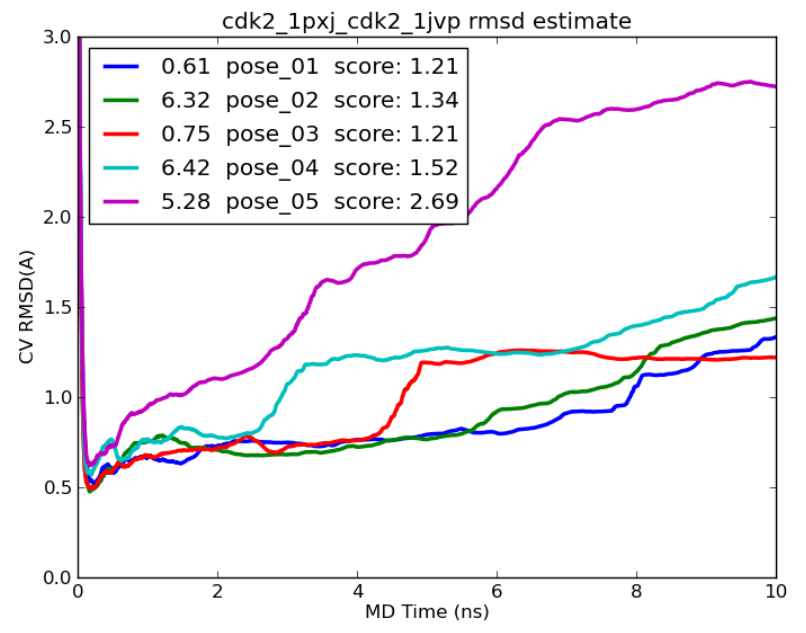

case 7
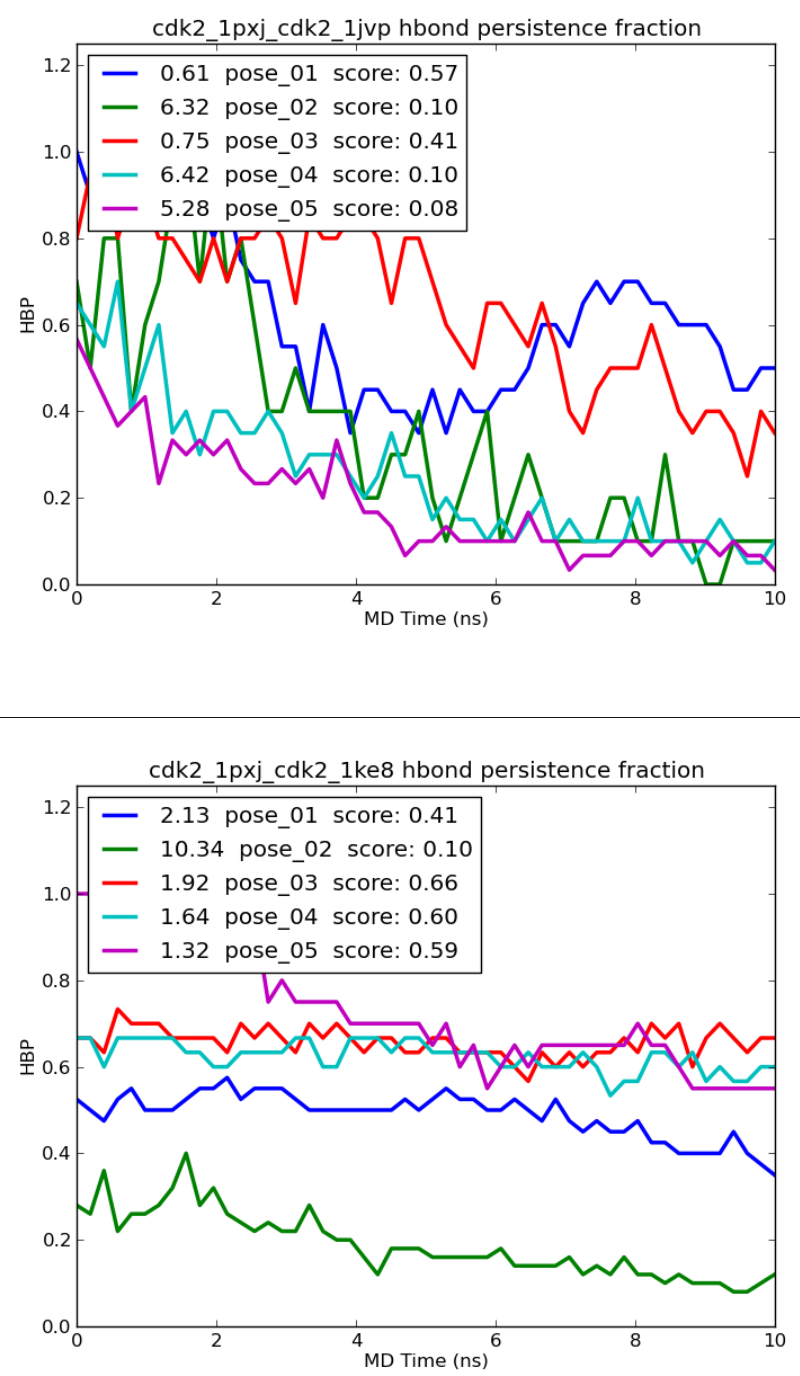

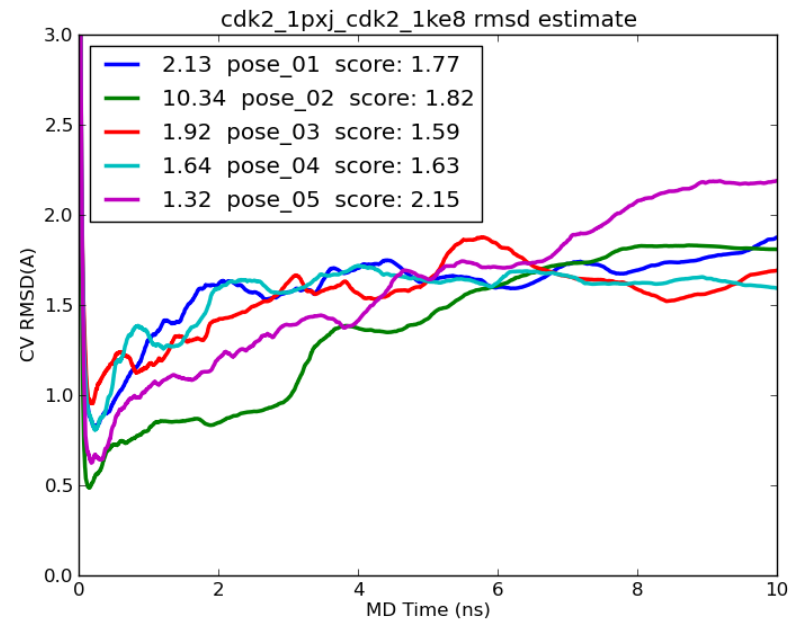

case 8
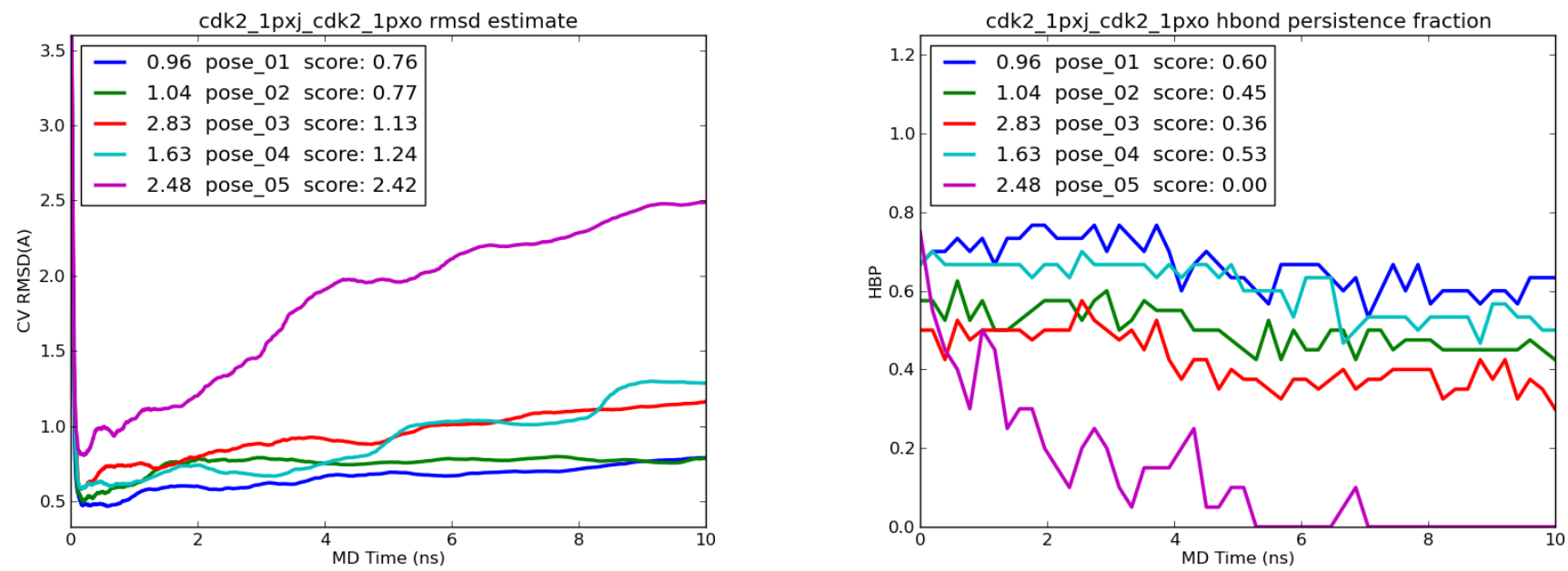

case 9 

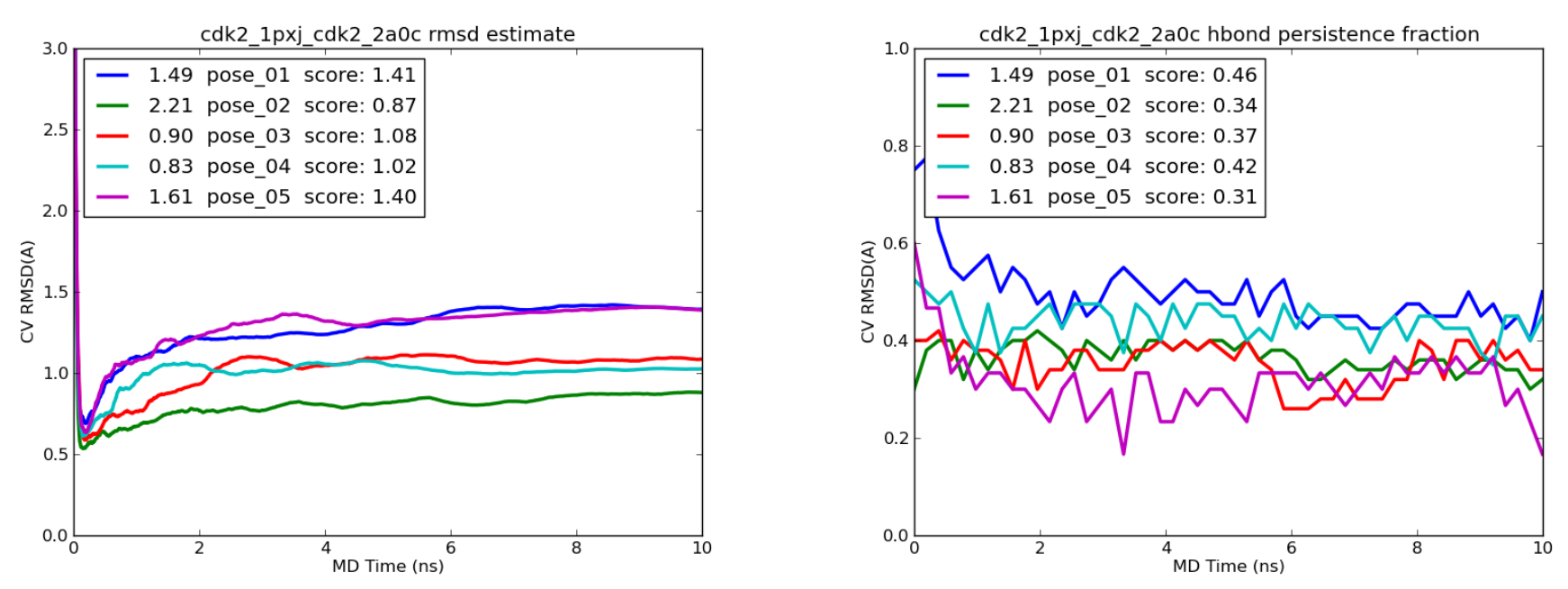

case 10

cdk2 1pxj cdk2 2btr rmsd estimate

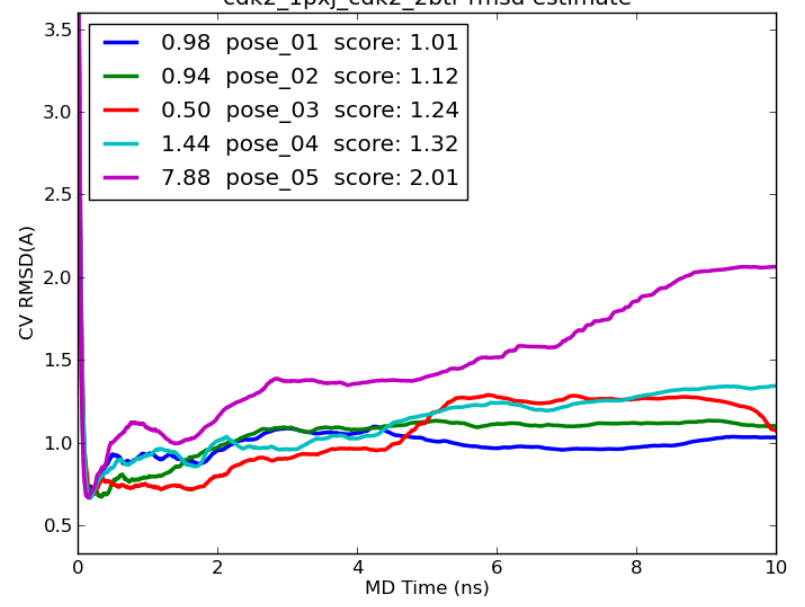

case 11
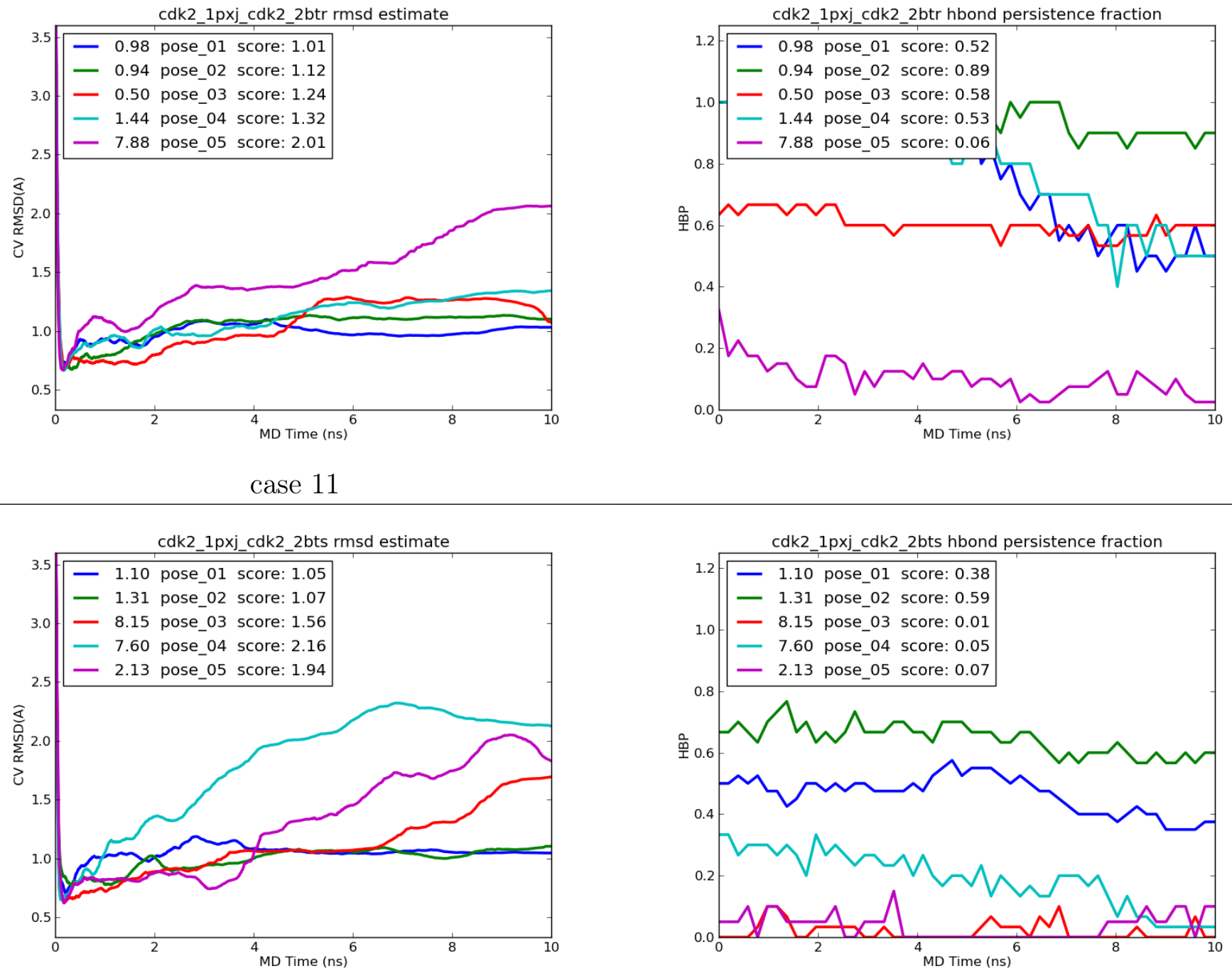

case 12 
cdk2_1pxj_cdk2_2c6m rmsd estimate

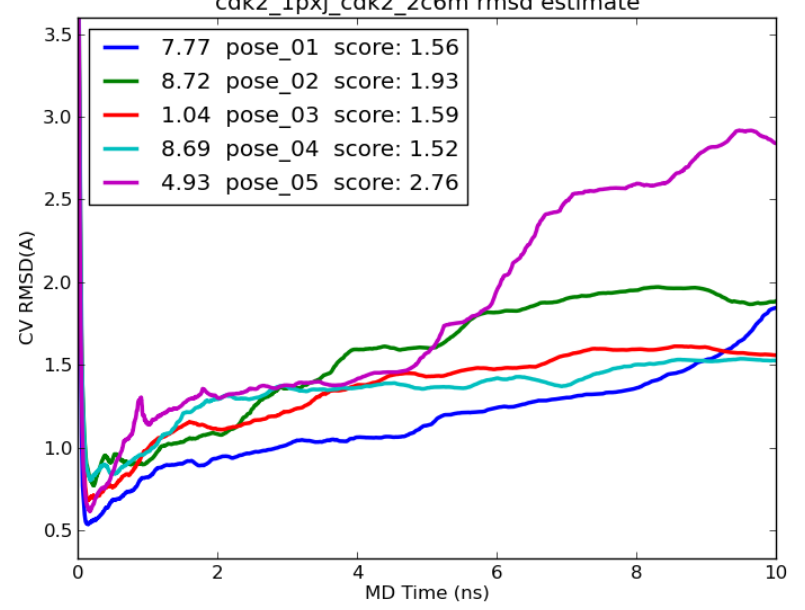

case 13
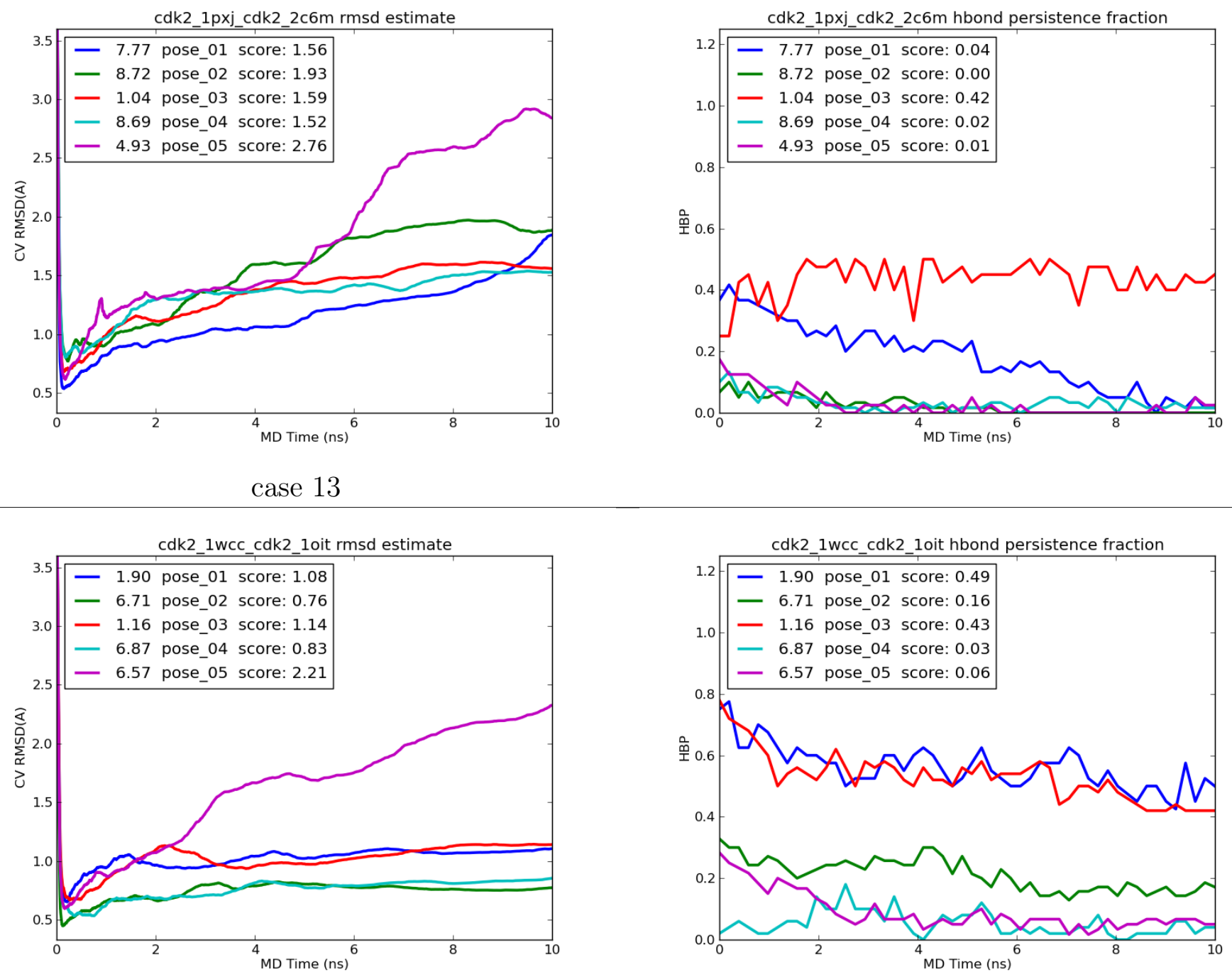

case 14
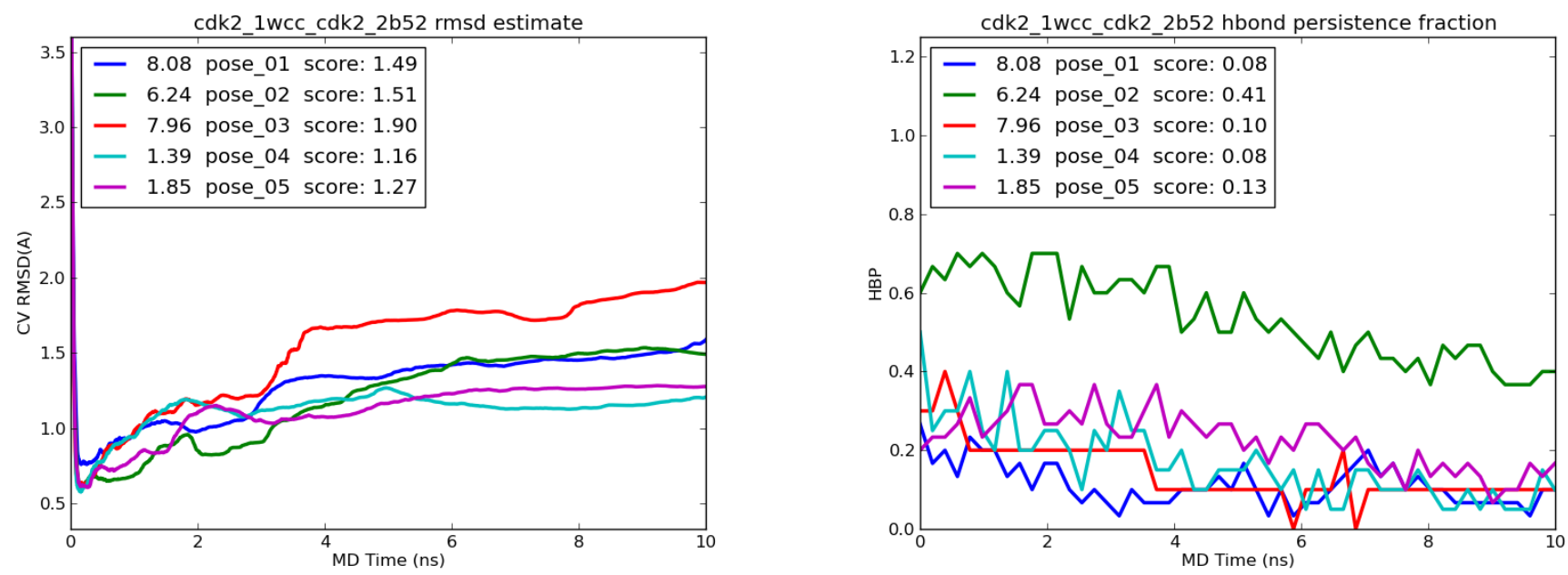

case 15 
cdk2 1wcc cdk2 2 bhe rmsd estimate

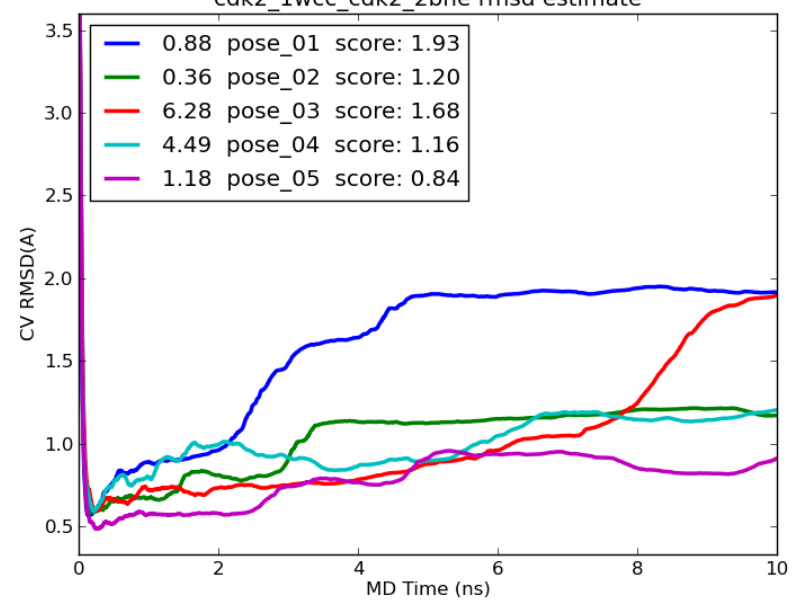

case 16
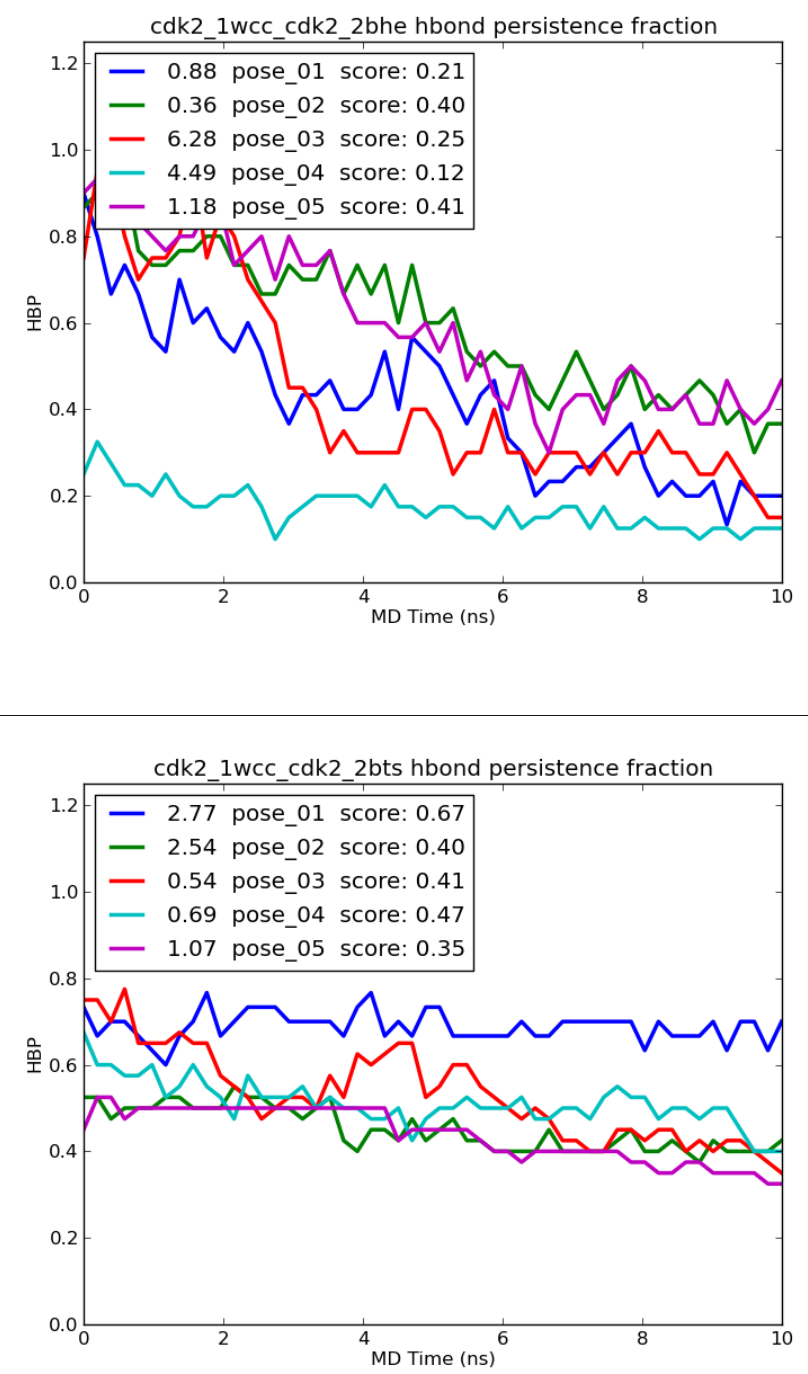

case 17
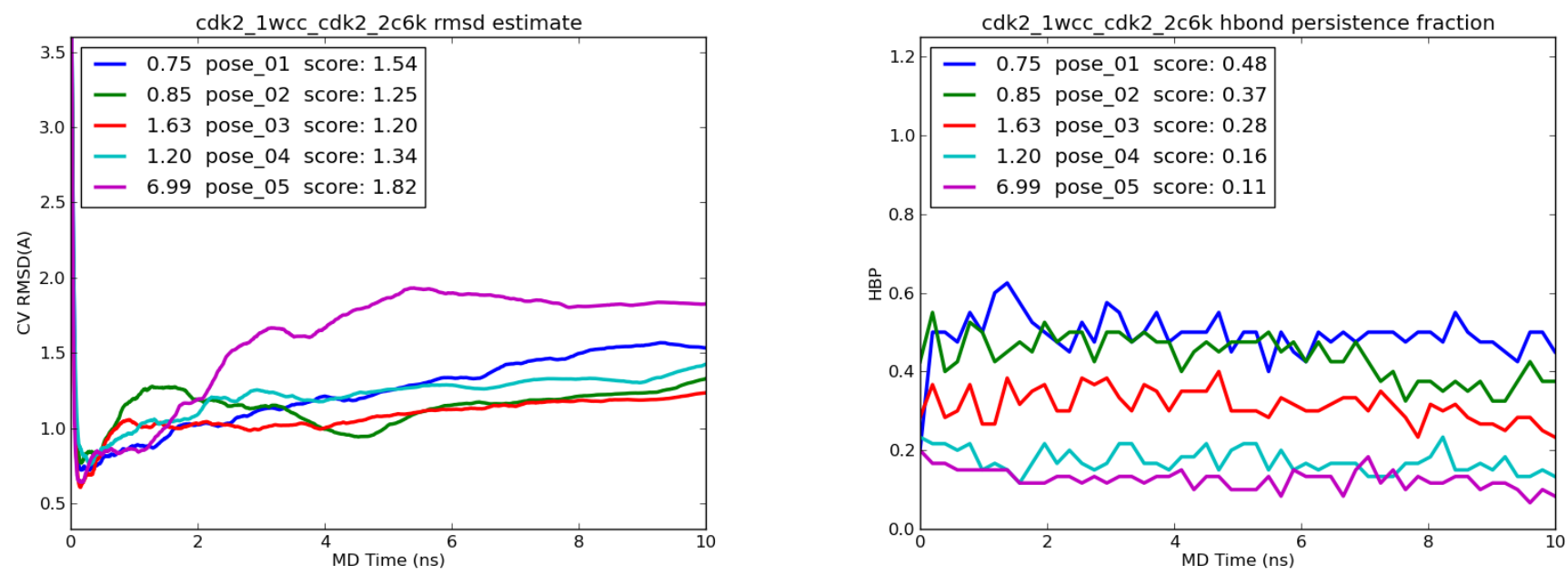

case 18 
chk1_2c3j_chk1_1nvs rmsd estimate

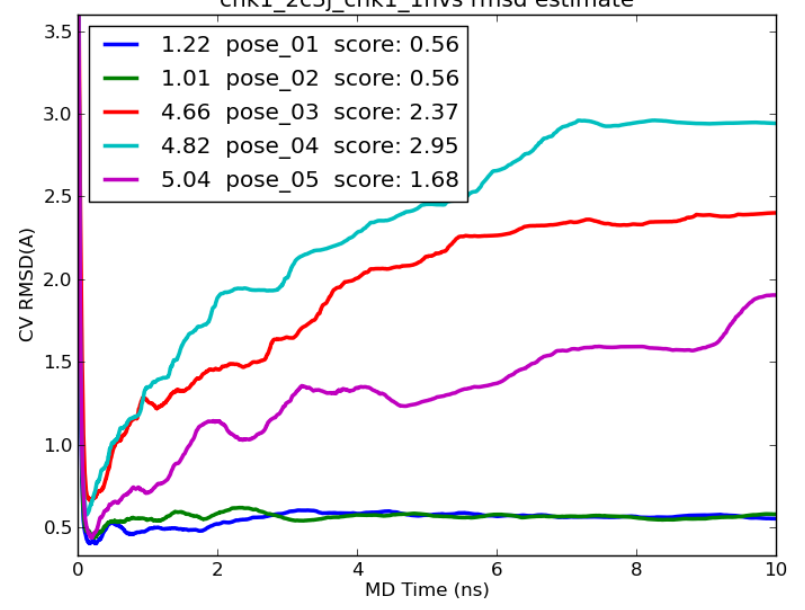

case 19

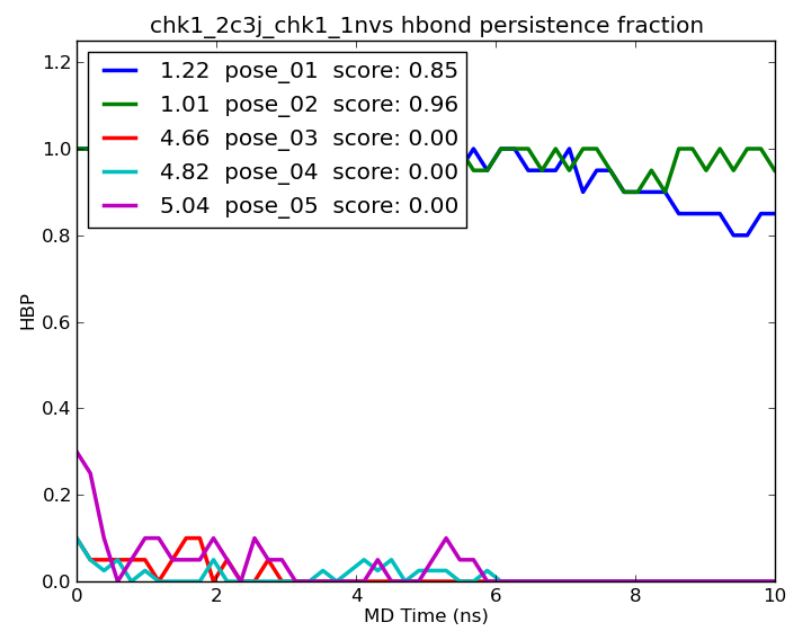

chk1_2c3j_chk1_2cgu rmsd estimate

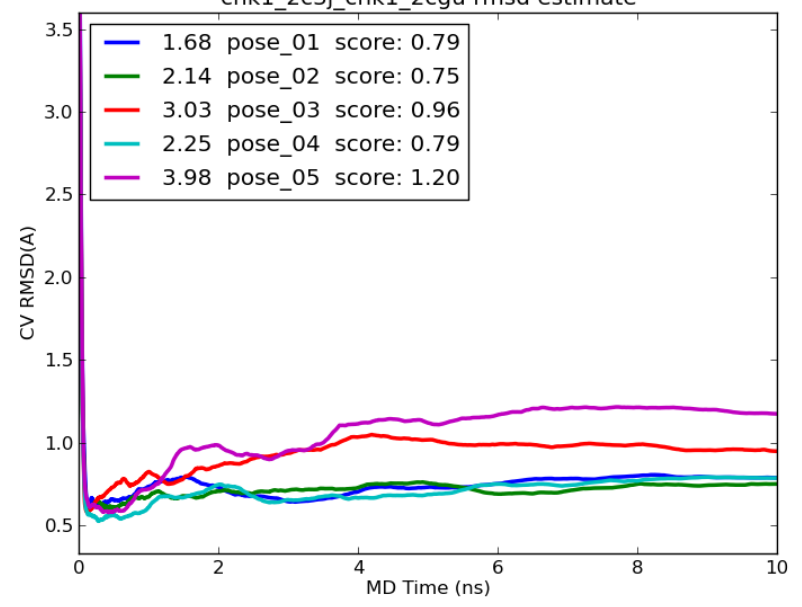

case 20
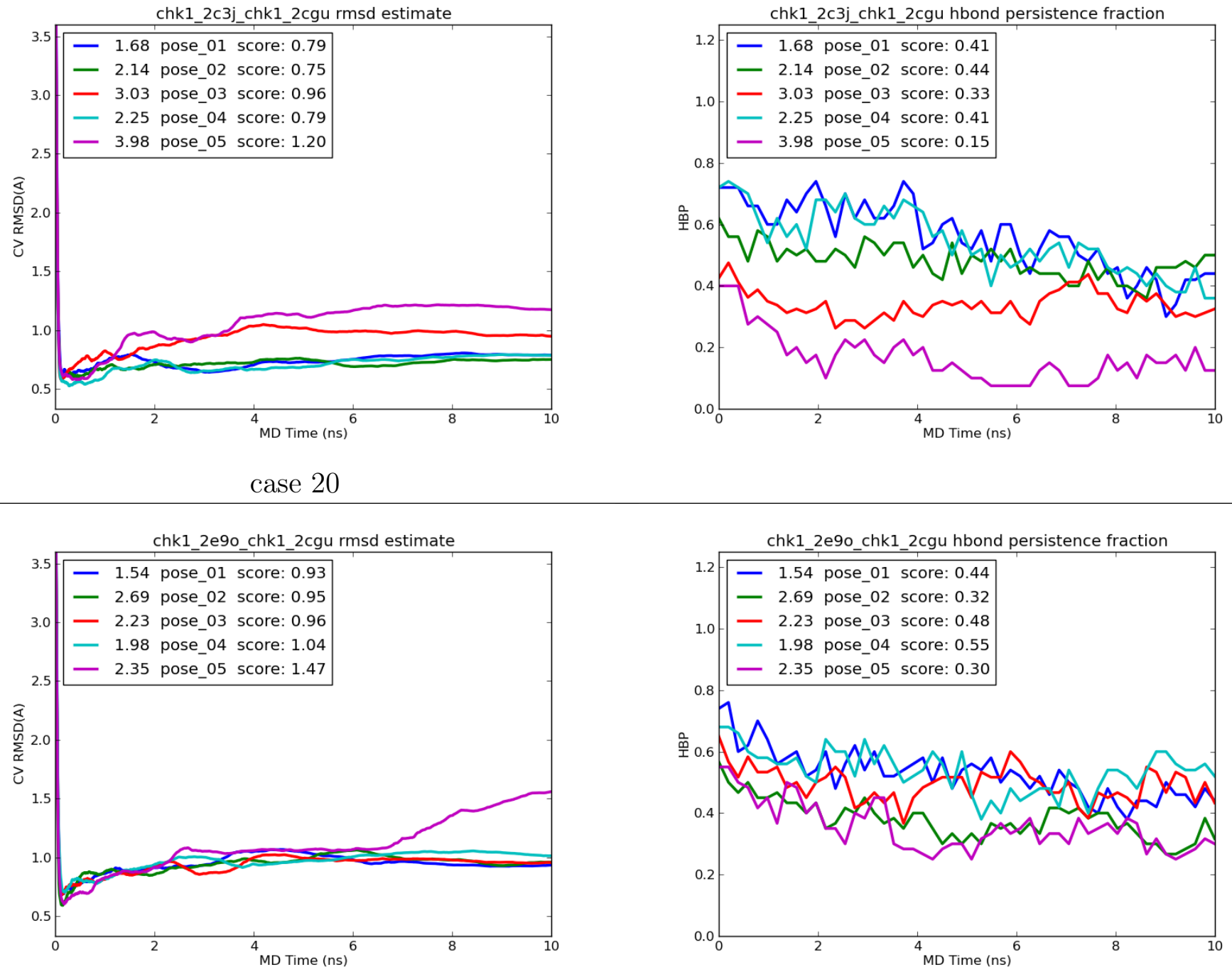

case 21 
chk1_2e9o_chk1_2cgx rmsd estimate

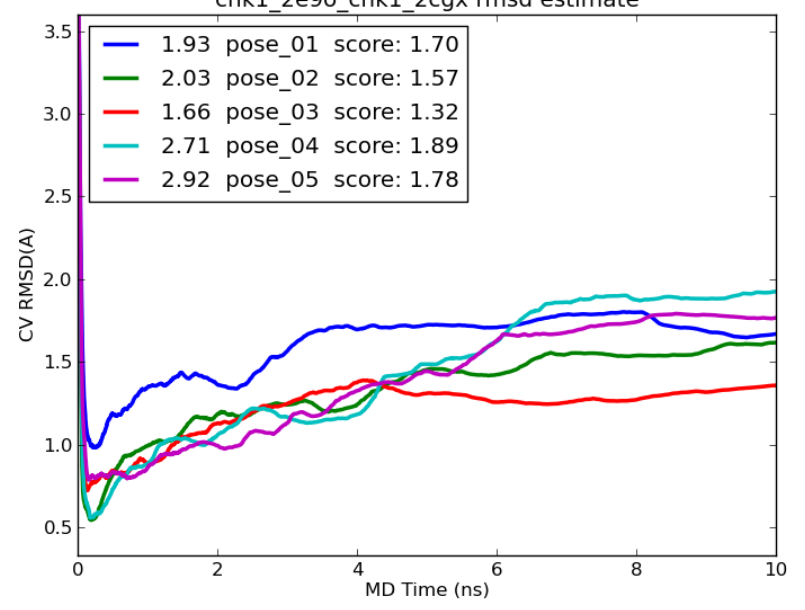

case 22
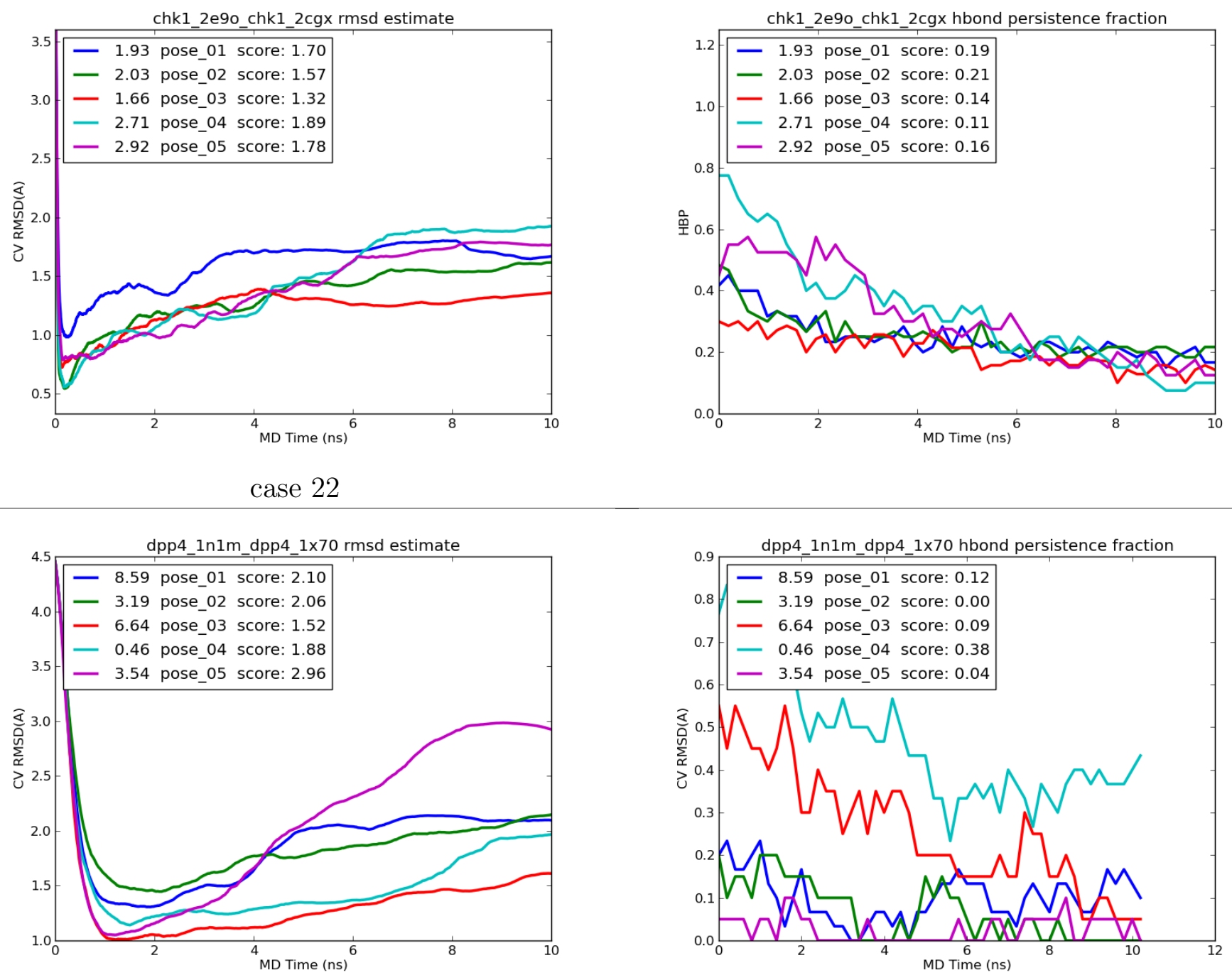

case 23
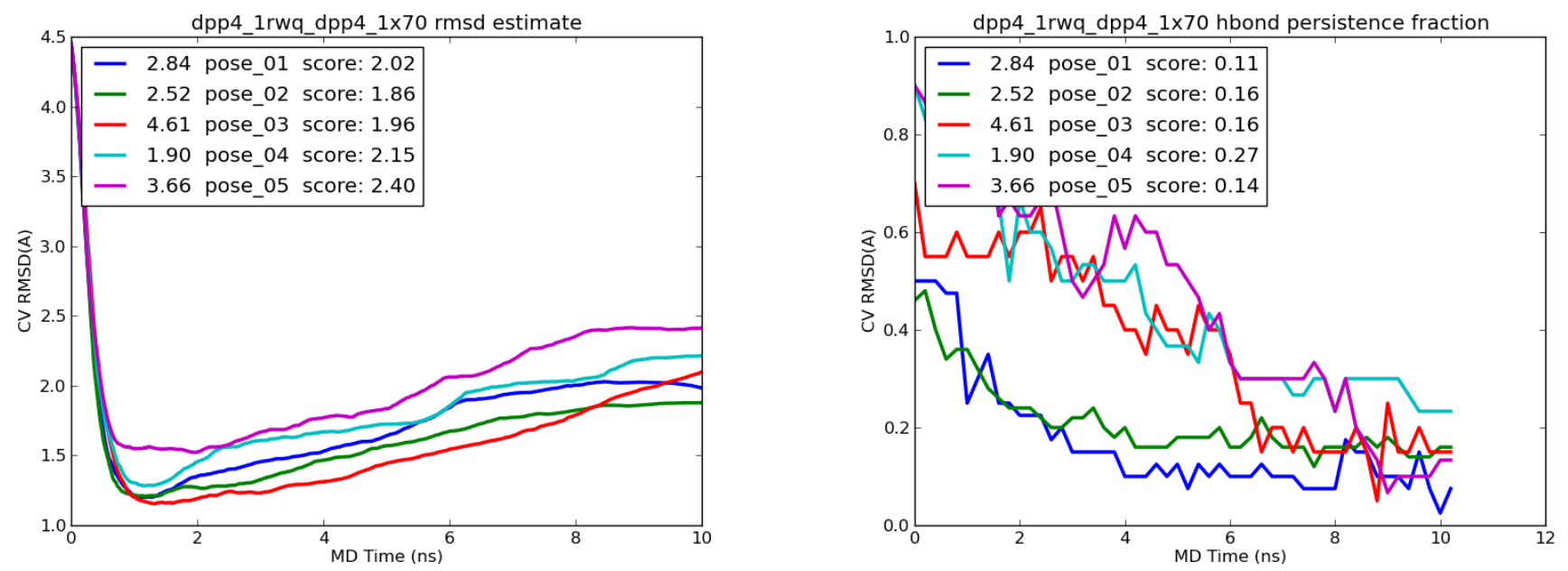

case 24 
fxa 1g2m fxa 1lpk rmsd estimate

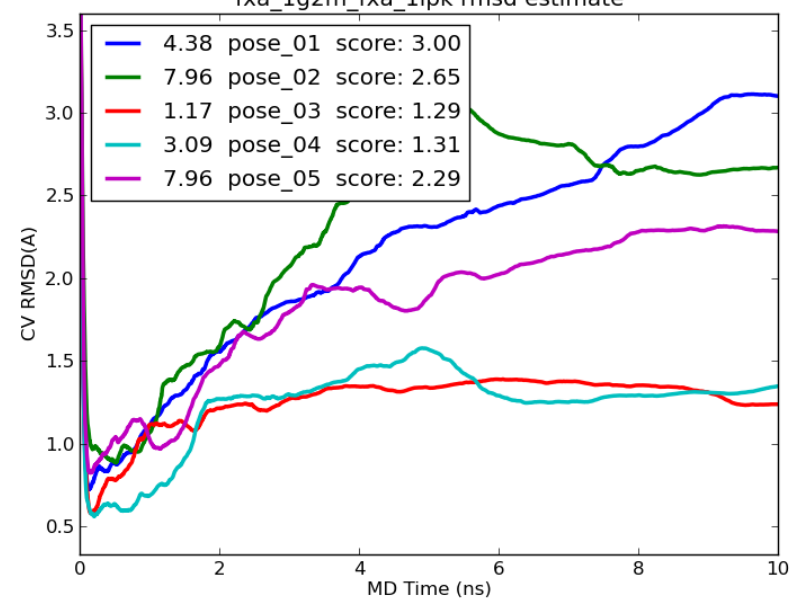

case 25

fxa Infx fxa 1lpk rmsd estimate

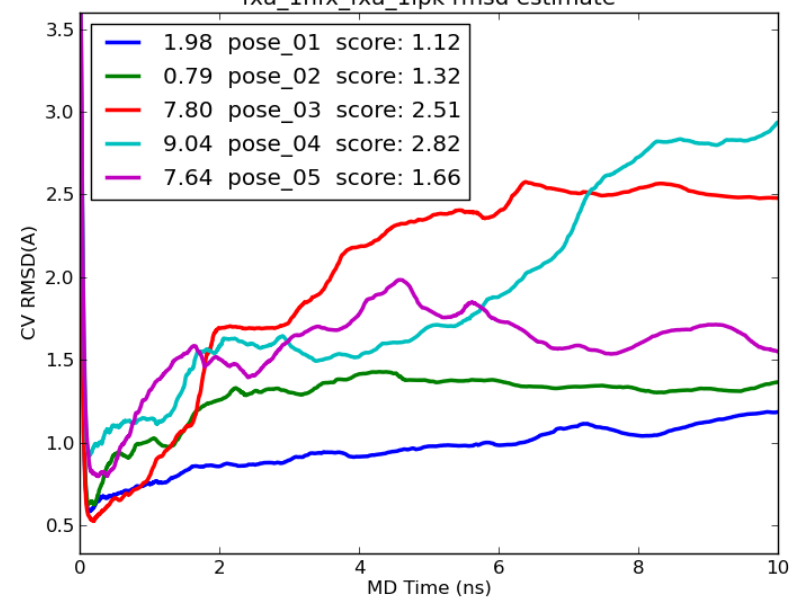

case 26
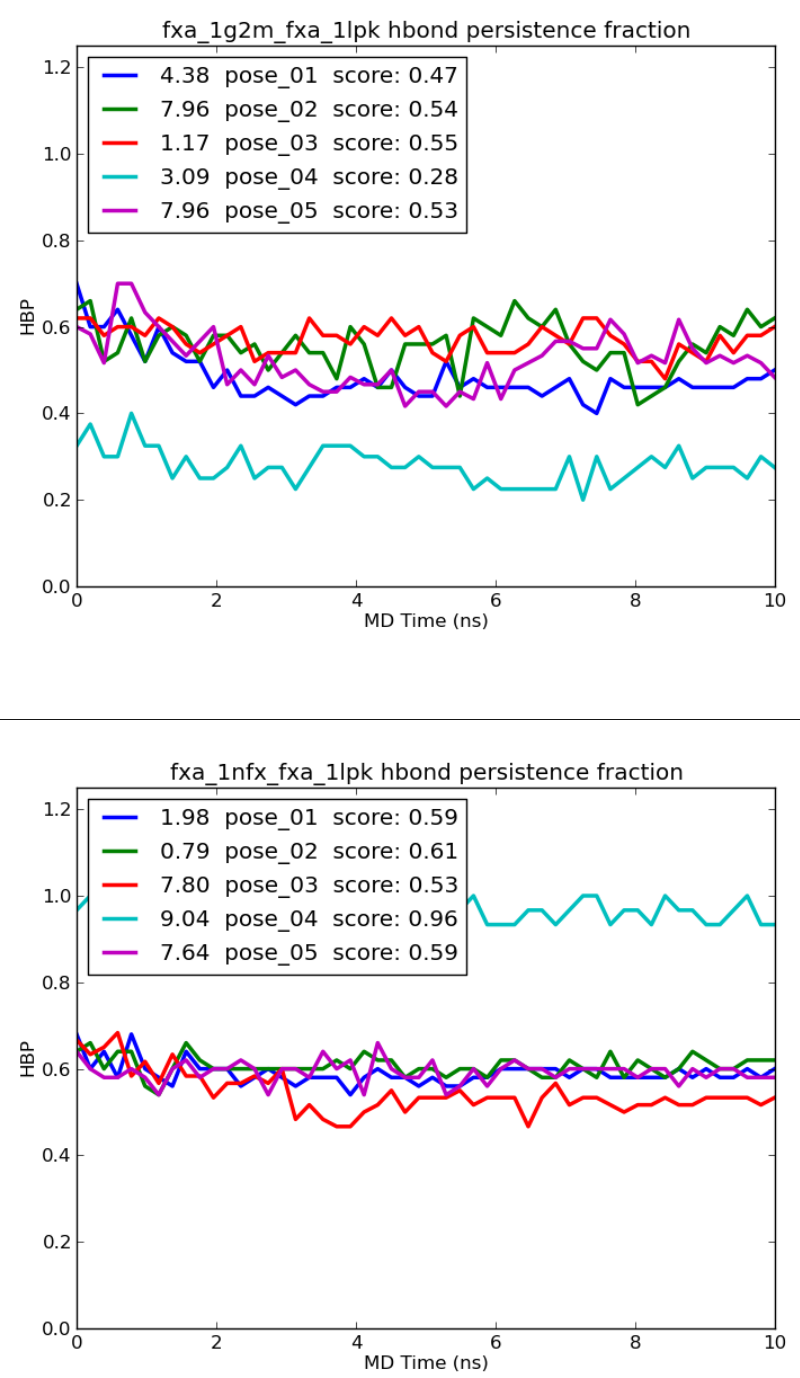
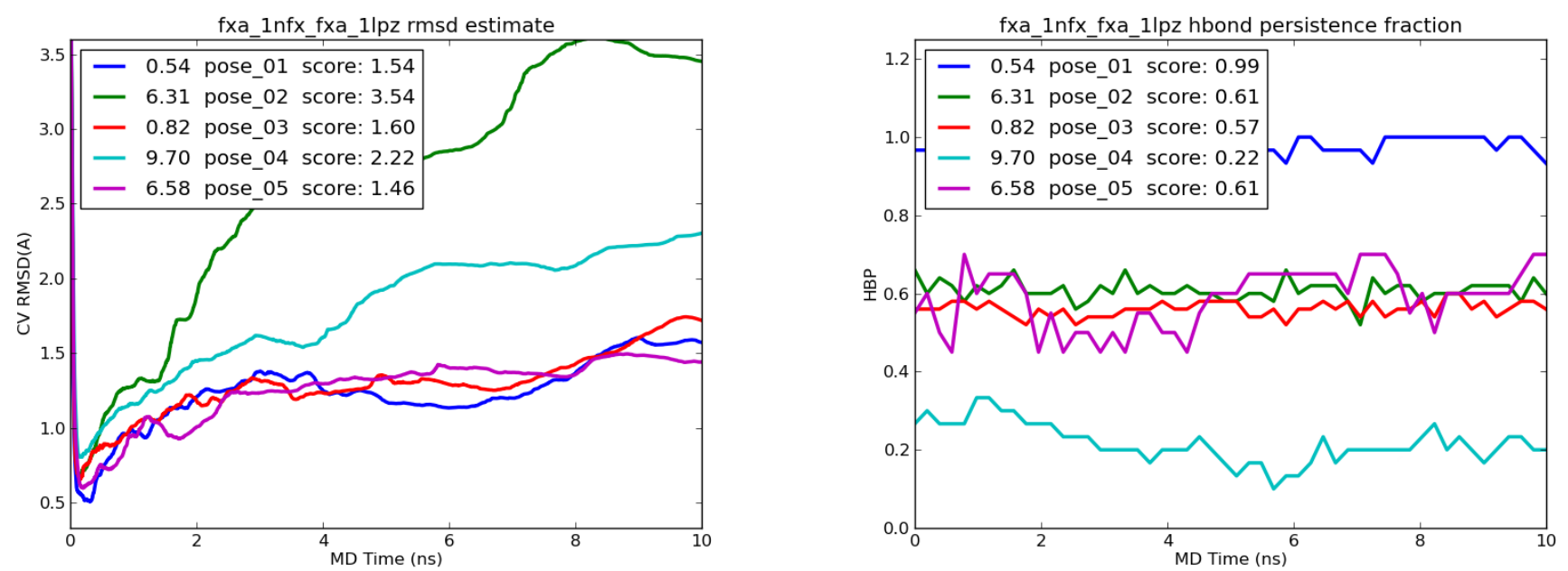

case 27 
fxa Infx fxa llad rmsd estimate

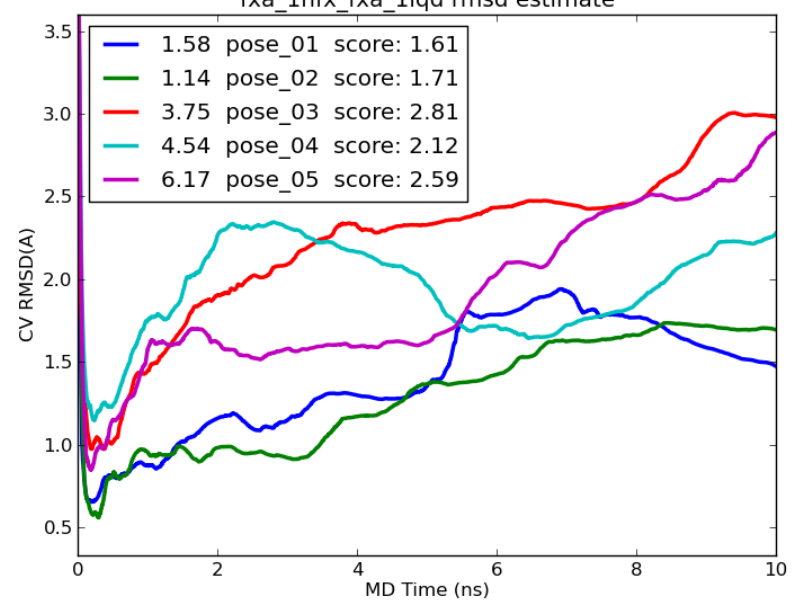

fxa Infx fxa 1lqd hbond persistence fraction

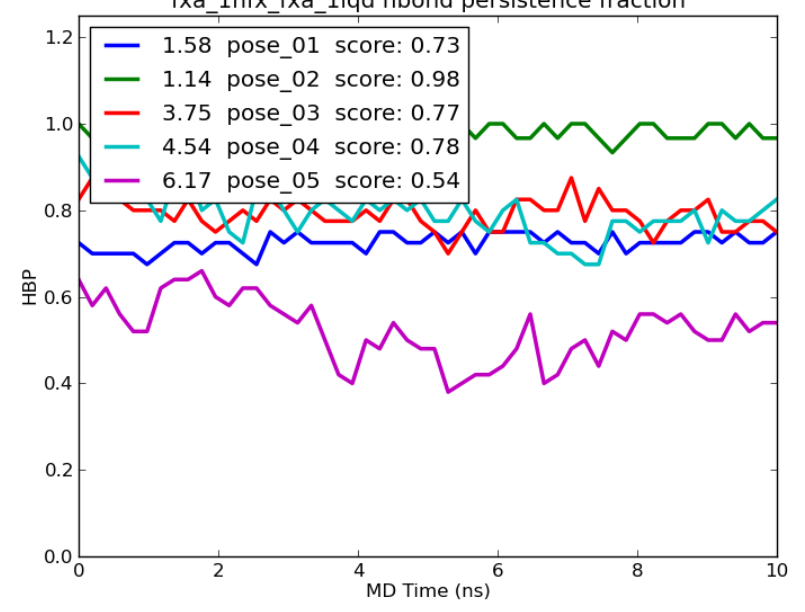

case 28
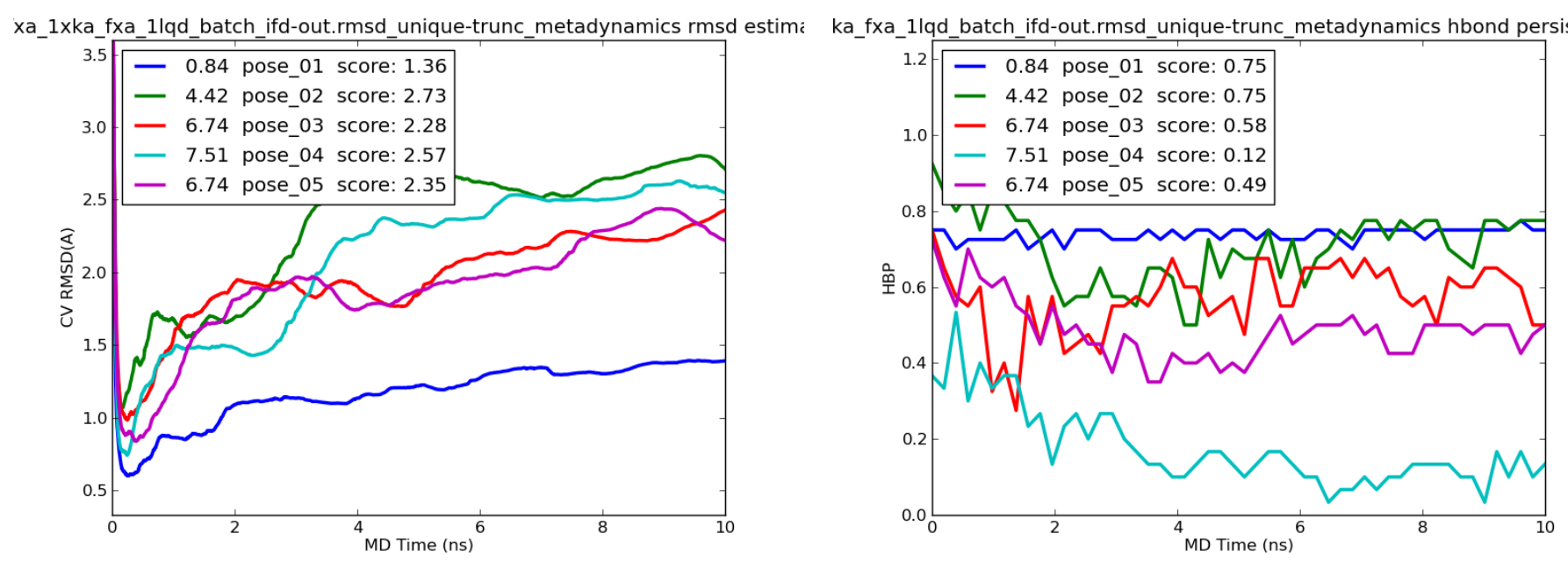

case 29
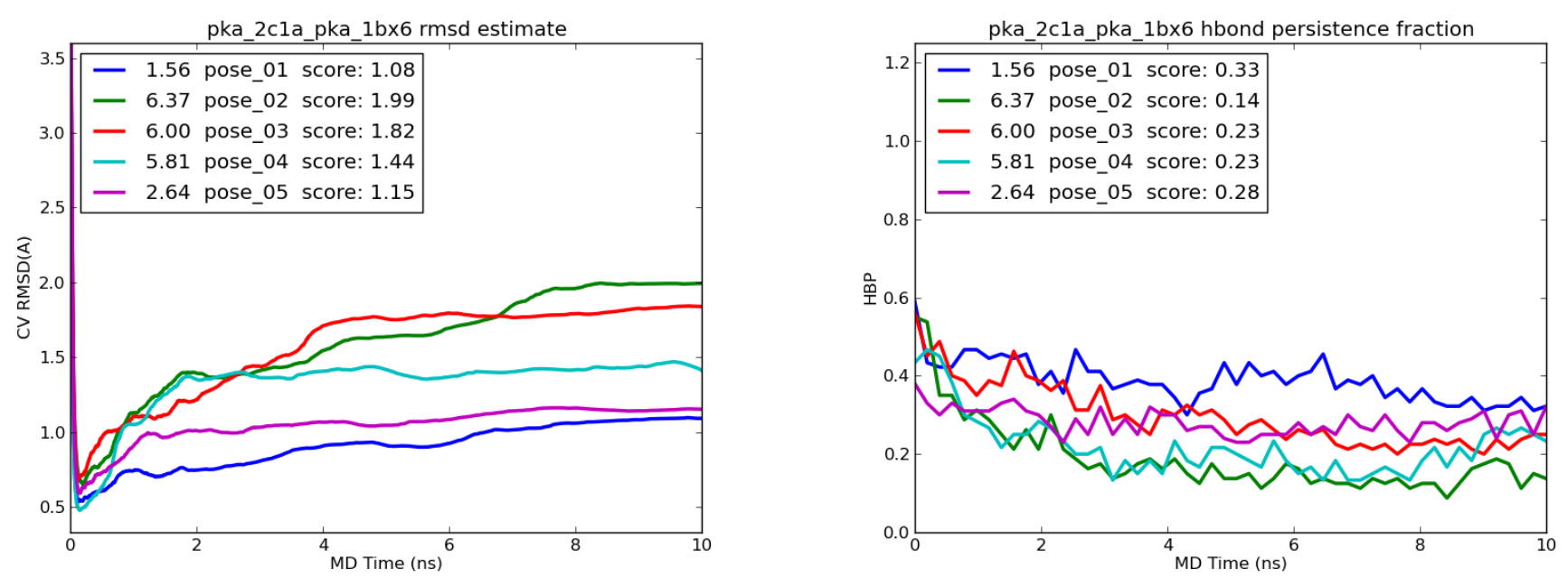

case 30 
pka_2erz pka 1cdk rmsd estimate

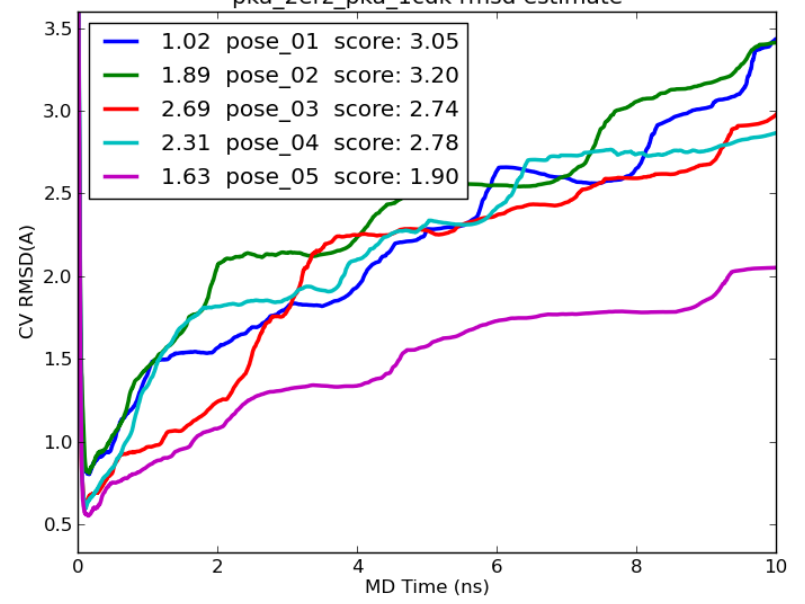

case 31

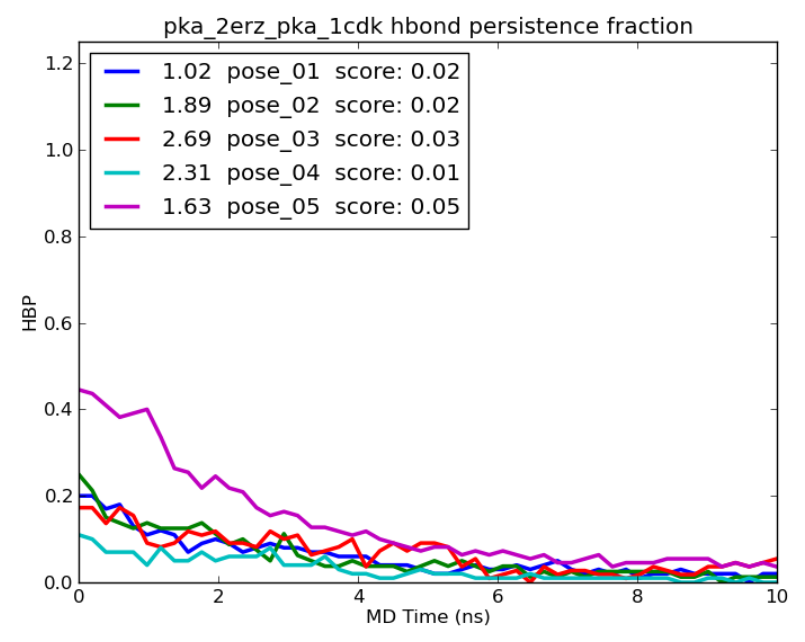

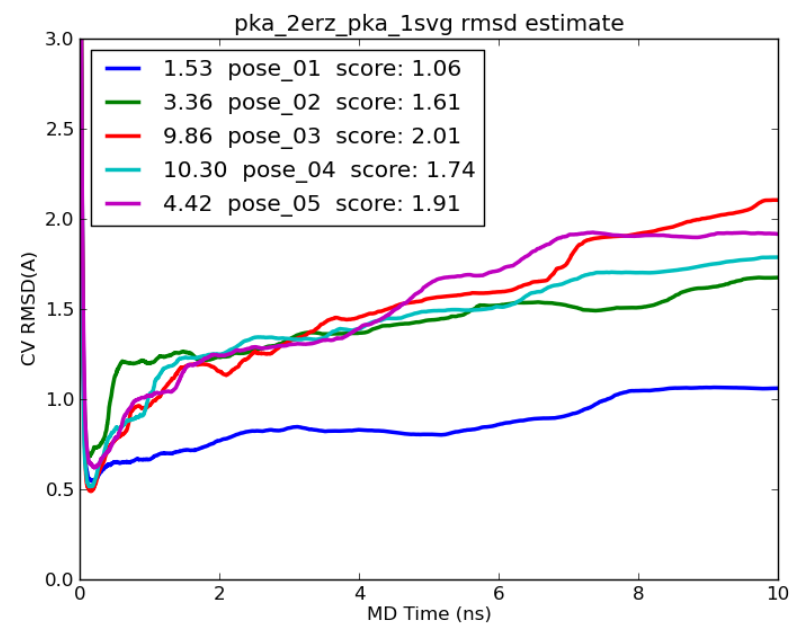

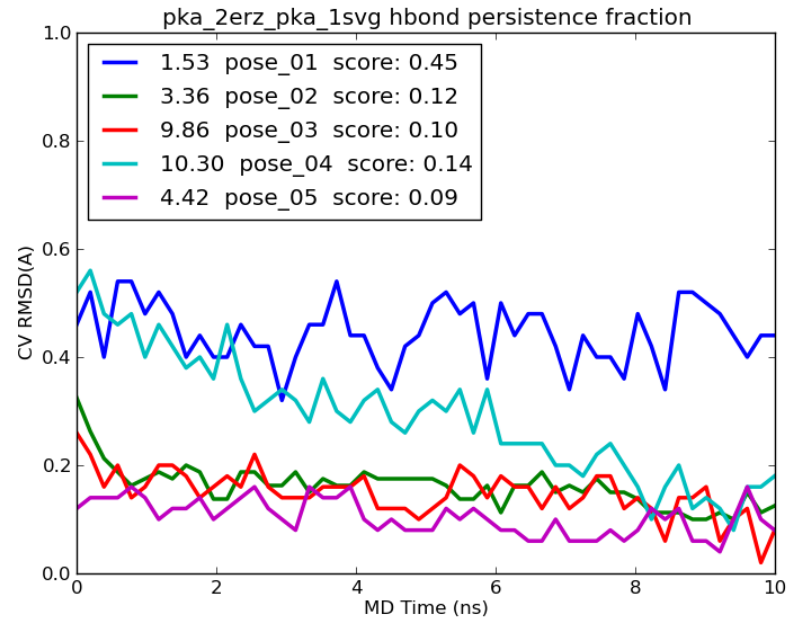

case 32
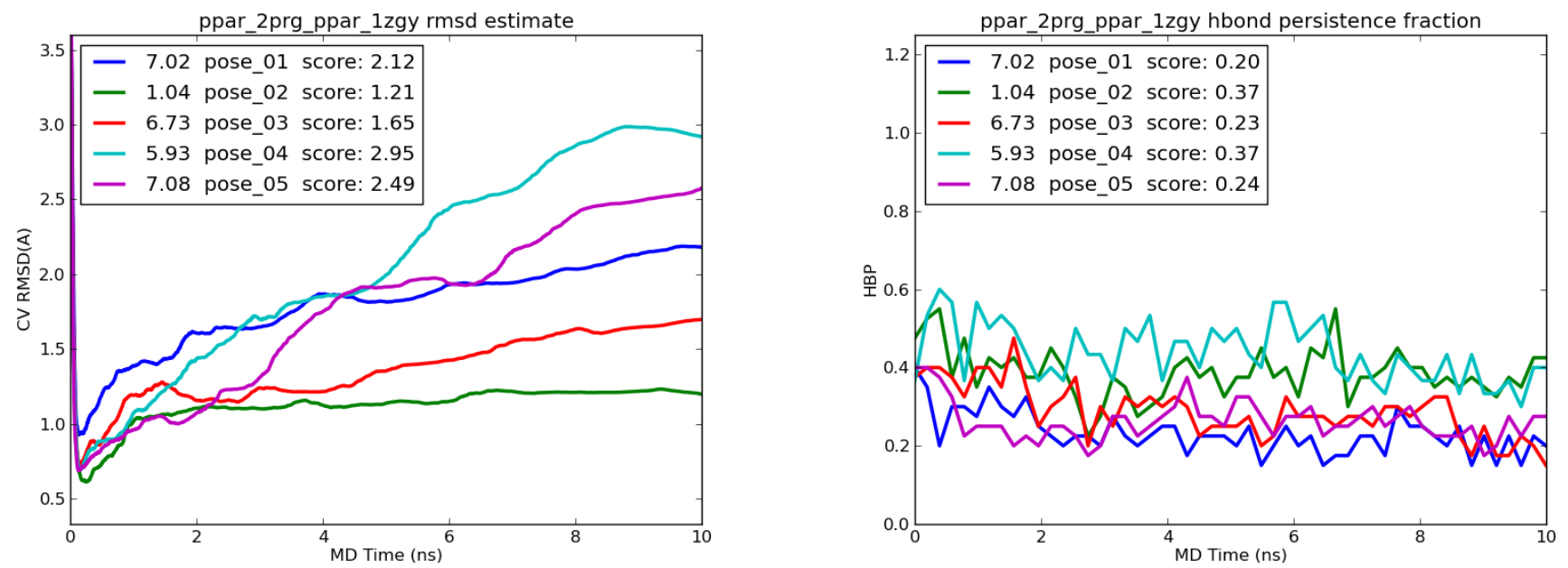

case 33 
ppar_2prg ppar 2ath rmsd estimate

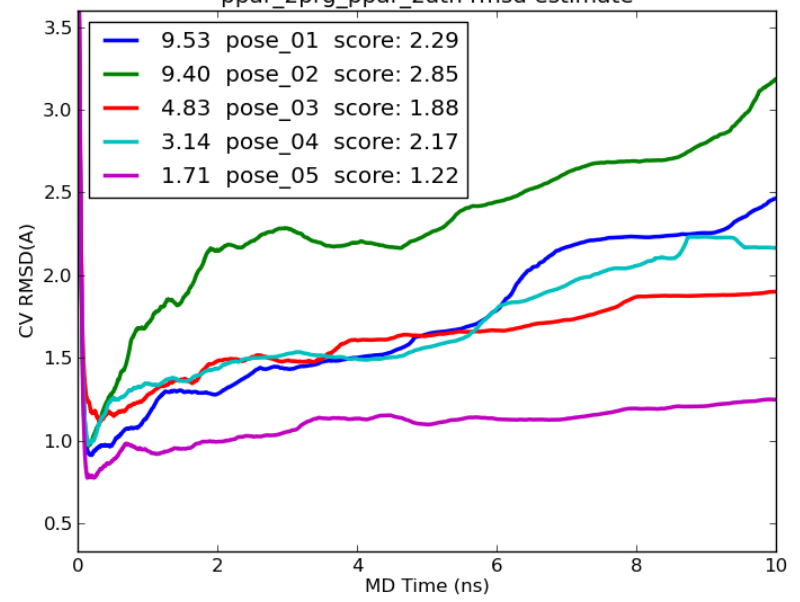

case 34
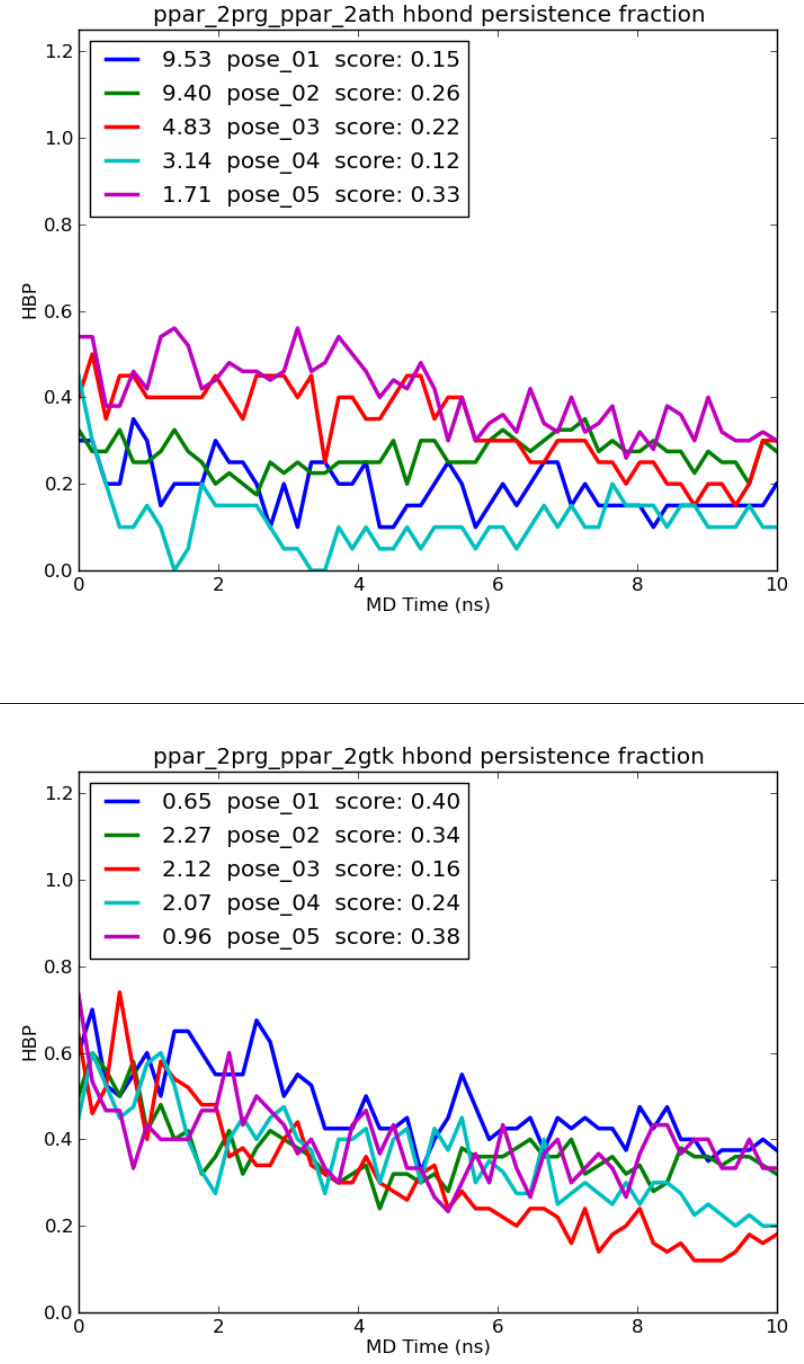

case 35
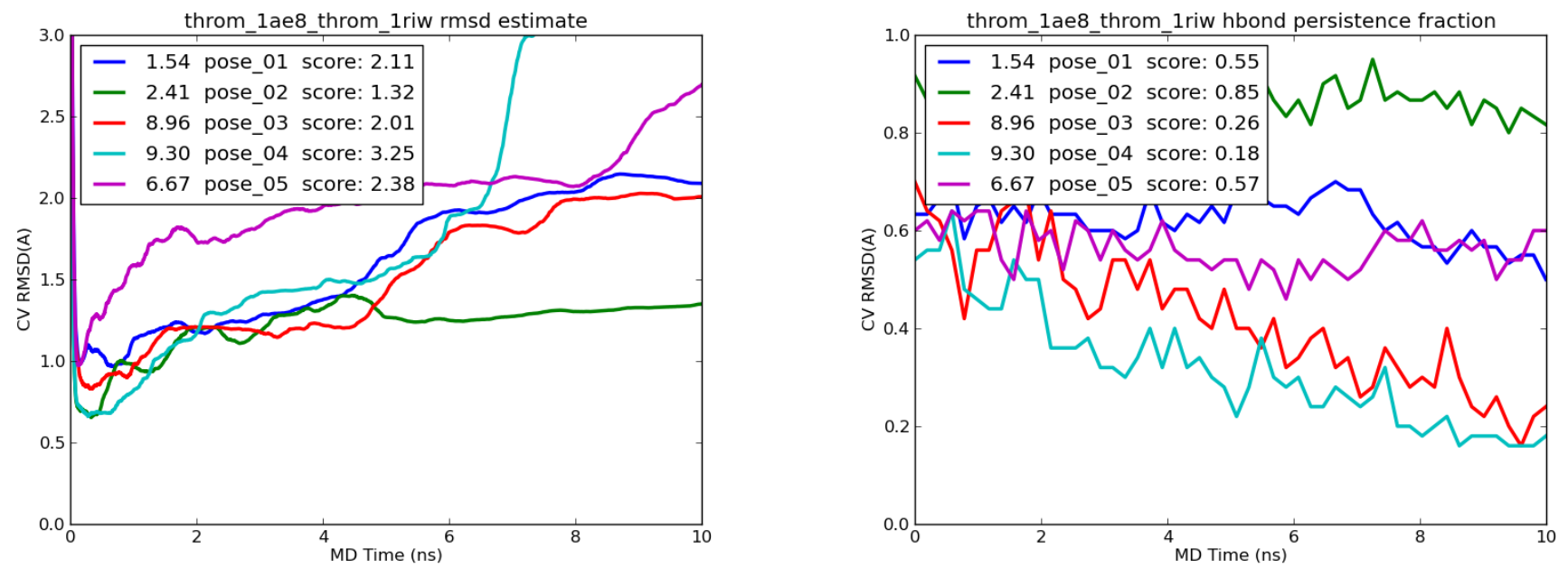

case 36 
throm 1 k21 throm 1riw rmsd estimate

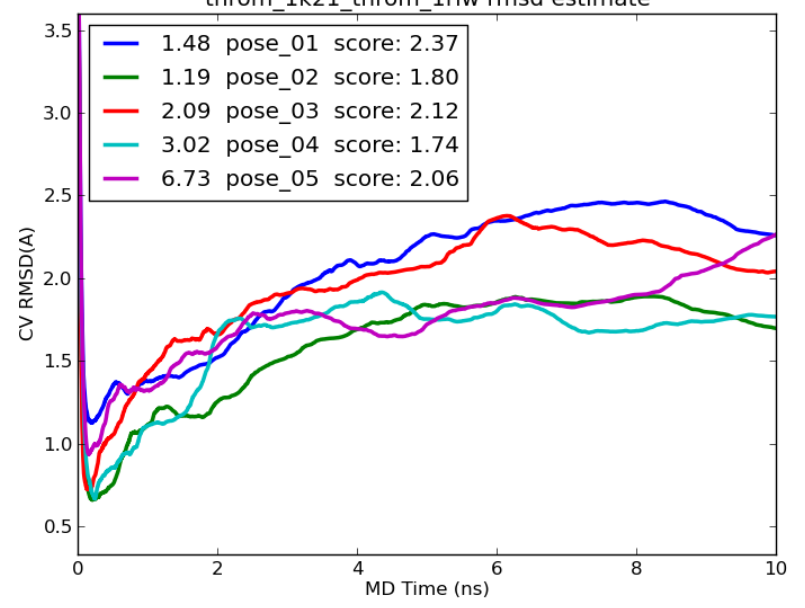

case 37
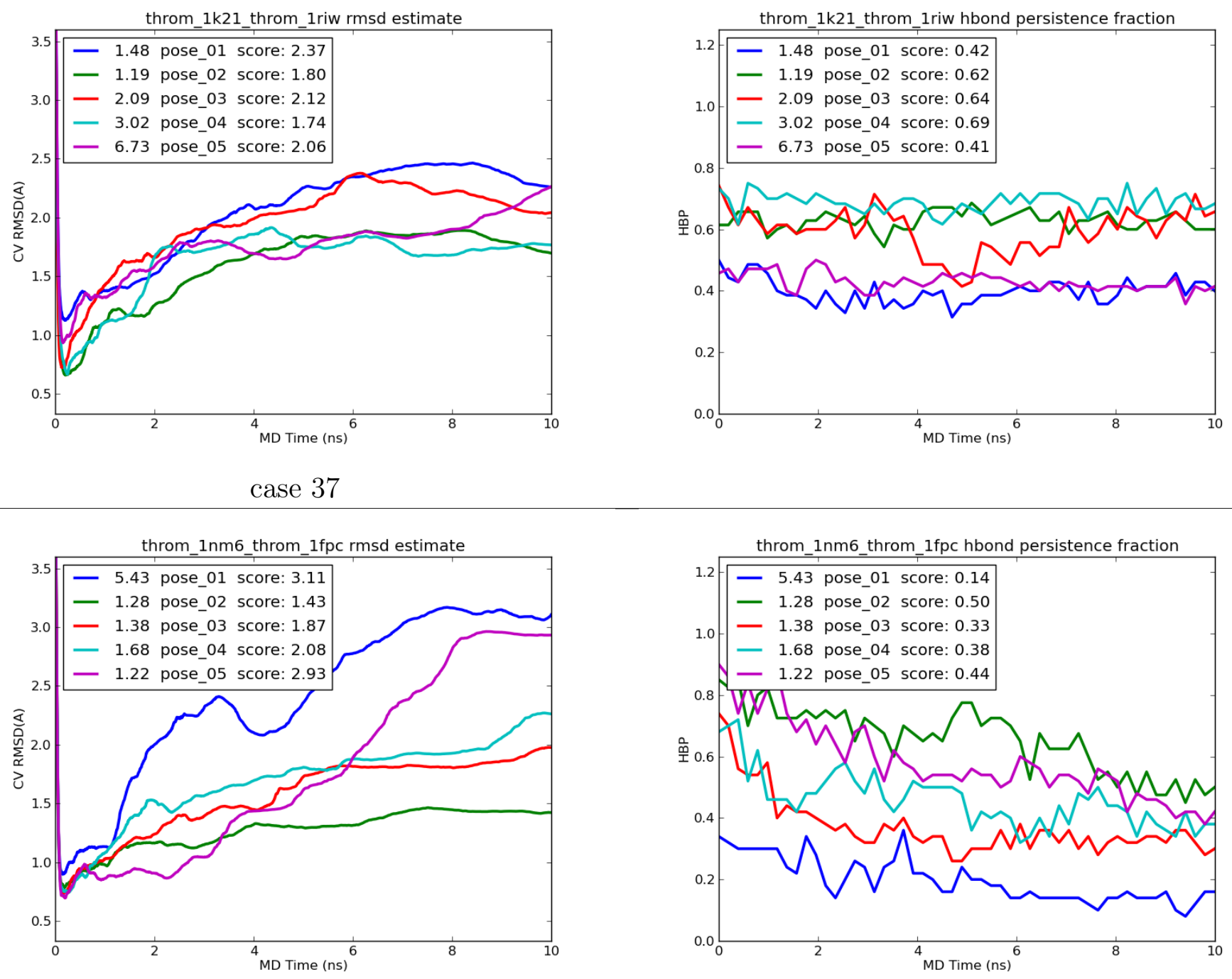

case 38
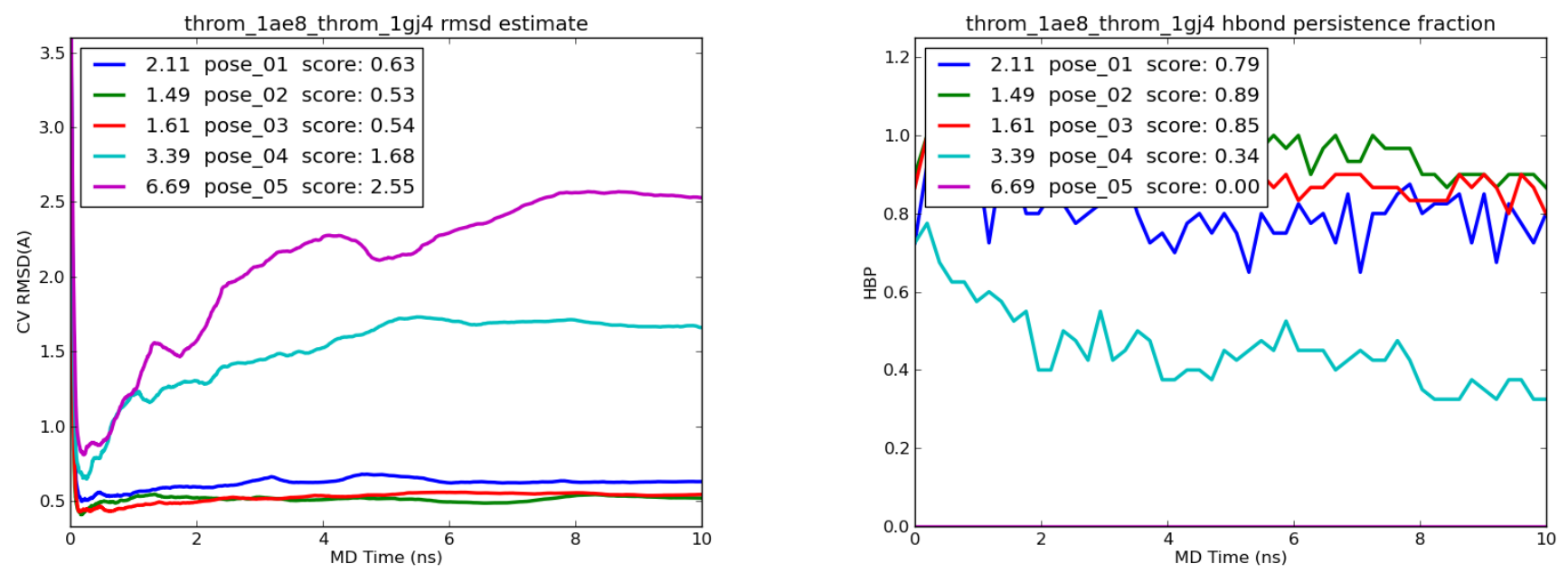

case 39 
throm 1ae8 throm 1gj5 rmsd estimate

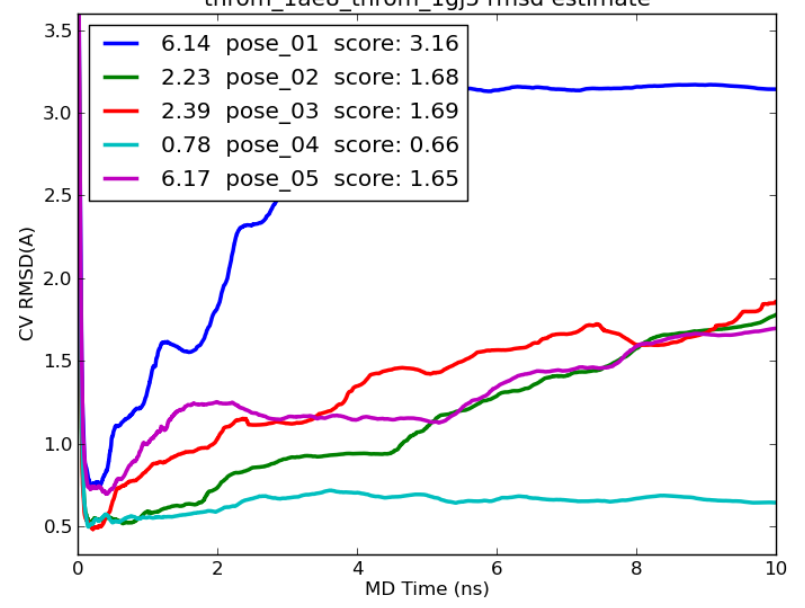

case 40
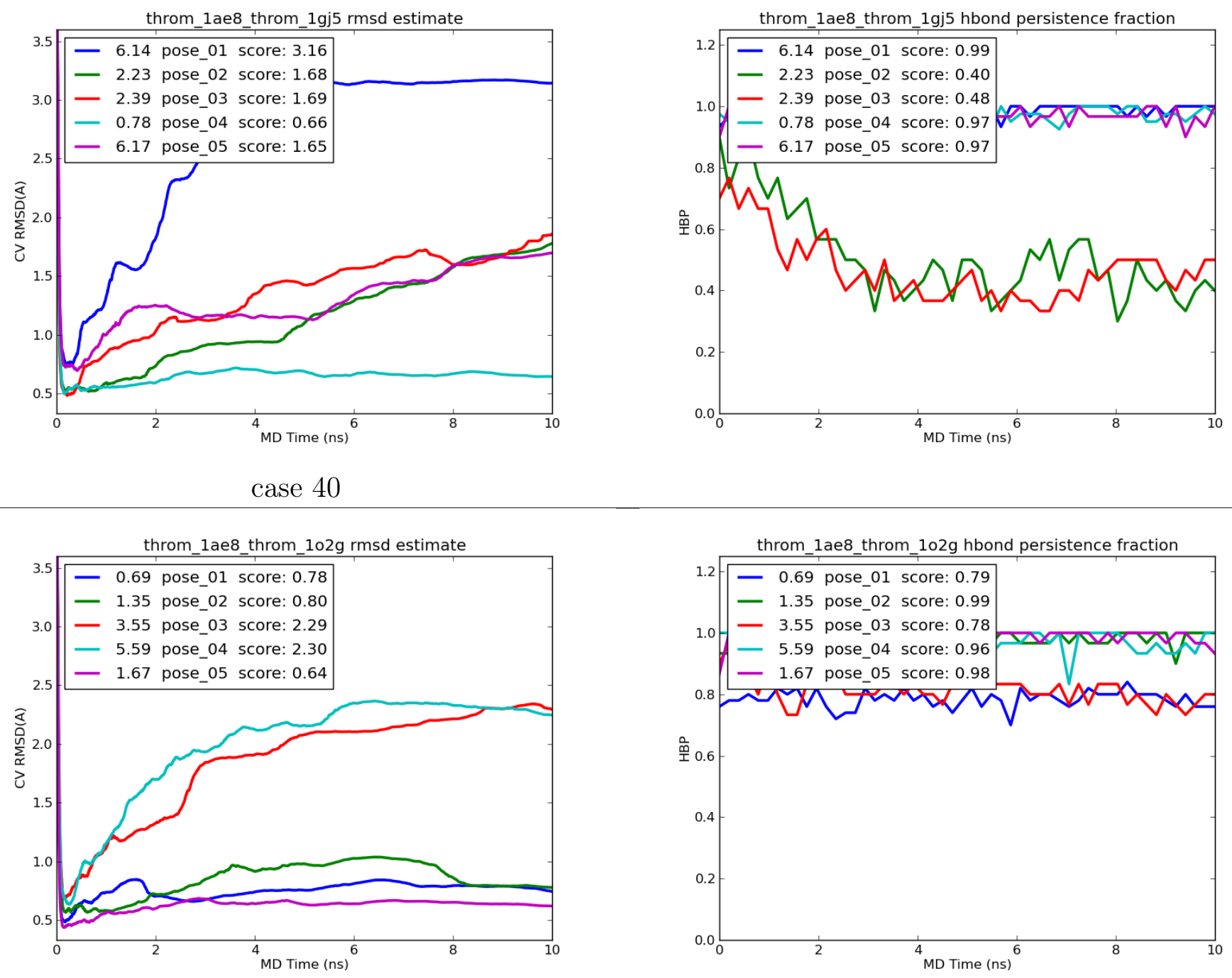

case 41
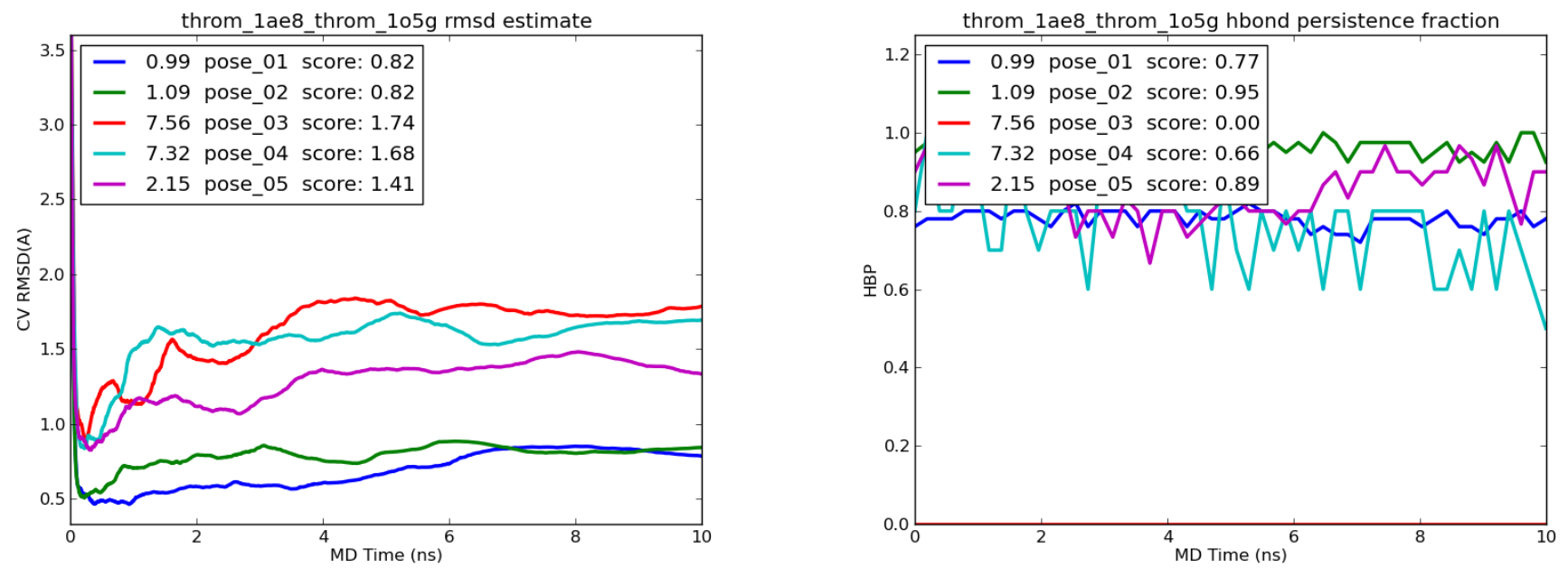

case 42 